\title{
THE TRANSITIONAL STRIPPED-ENVELOPE SN 2008ax: SPECTRAL EVOLUTION AND EVIDENCE FOR LARGE ASPHERICITY
}

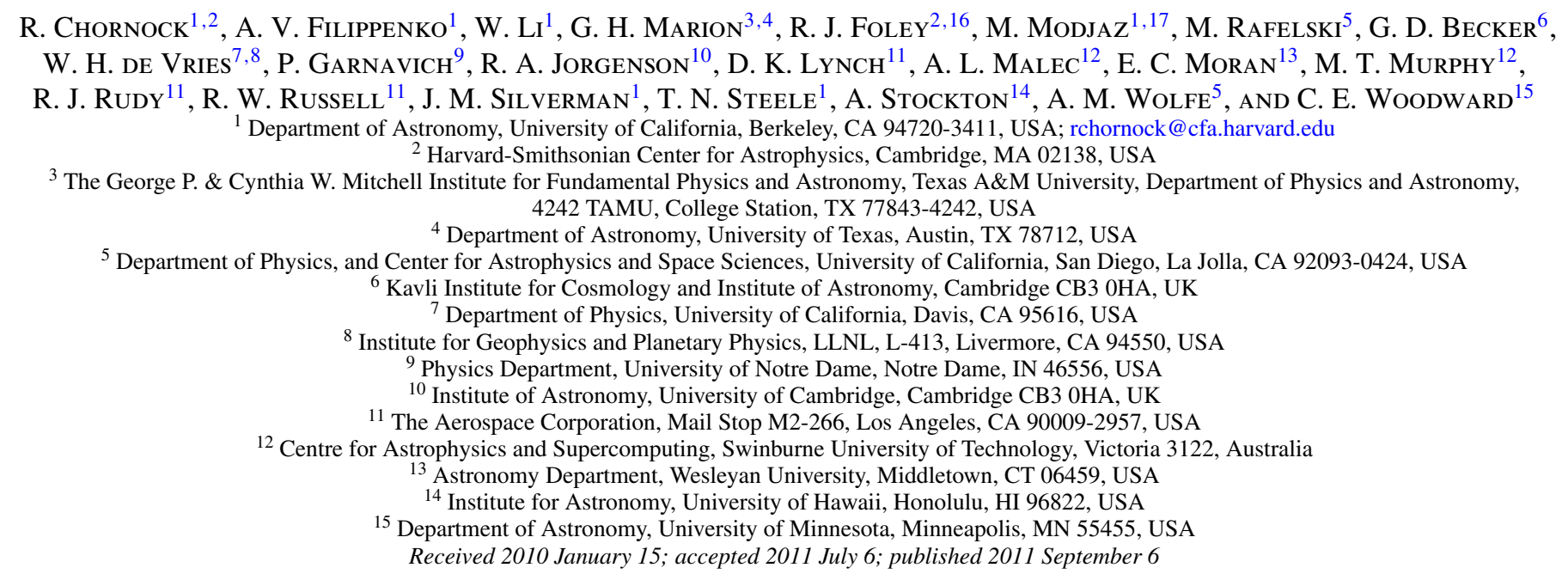

\begin{abstract}
Supernova (SN) 2008ax in NGC 4490 was discovered within hours after shock breakout, presenting the rare opportunity to study a core-collapse SN beginning with the initial envelope-cooling phase immediately following shock breakout. We present an extensive sequence of optical and near-infrared spectra, as well as three epochs of optical spectropolarimetry. Our initial spectra, taken two days after shock breakout, are dominated by hydrogen Balmer lines at high velocity. However, by maximum light, He I lines dominated the optical and near-infrared spectra, which closely resembled those of normal Type Ib supernovae (SNe Ib) such as SN 1999ex. This spectroscopic transition defines Type IIb SNe, but the strong similarity of SN 2008ax to normal SNe Ib beginning near maximum light, including an absorption feature near $6270 \AA$ due to $\mathrm{H} \alpha$ at high velocities, suggests that many objects classified as SNe Ib in the literature may have ejected similar amounts of hydrogen as SN 2008ax, roughly a few $\times 0.01 M_{\odot}$. Only the unusually early discovery of SN 2008ax allowed us to observe the spectroscopic signatures of the hydrogen-rich outer ejecta. Early-time spectropolarimetry (six and nine days after shock breakout) revealed strong line polarization modulations of $3.4 \%$ across $\mathrm{H} \alpha$, indicating the presence of large asphericities in the outer ejecta and possibly that the spectrum of SN 2008ax could be dependent on the viewing angle. After removal of interstellar polarization, the continuum shares a common polarization angle with the hydrogen, helium, and oxygen lines, while the calcium and iron absorptions are oriented at different angles. This is clear evidence of deviations from axisymmetry even in the outer ejecta. Intrinsic continuum polarization of $0.64 \%$ only nine days after shock breakout shows that the outer layers of the ejecta were quite aspherical. A single epoch of late-time spectropolarimetry as well as the shapes of the nebular line profiles demonstrate that asphericities extended from the outermost layers all the way down to the center of this core-collapse SN. SN 2008ax may in some ways be an extragalactic analog of the explosion giving rise to Cassiopeia A, which has recently been determined to be a remnant of an SN IIb.
\end{abstract}

Key words: polarization - supernovae: general - supernovae: individual (SN 2008ax)

Online-only material: color figures

\section{INTRODUCTION}

Massive stars explode at the end of their lives as corecollapse supernovae ( $\mathrm{SNe}$ ), which we observe and classify spectroscopically on the basis of their optical spectra near maximum light (see Filippenko 1997 for a review). These phenomenological spectroscopic classes are related to the state of the progenitor star at the time of explosion (e.g., Heger et al. 2003). SNe coming from stars that retain most of their massive hydrogen envelopes until the time of explosion are

\footnotetext{
${ }^{16}$ Clay Fellow.

${ }^{17}$ Miller Fellow.
}

classified as Type II, while those lacking hydrogen due to either stellar winds or interaction with a companion star are classified as Types Ib and Ic. Progenitors with only small amounts of hydrogen remaining in their outer envelopes explode as $\mathrm{SNe} \mathrm{IIb}$, a classification named because their spectra transition from Type II at early times to Type Ib at late times.

$\mathrm{SNe}$ IIb are up to $\sim 11 \%$ of the core-collapse SN population in a distance-limited sample ( $\mathrm{Li}$ et al. 2011; Smith et al. 2011). Classical SNe Ib are a further 7\% of the core-collapse SN sample, although some of those objects (such as SN 1999dn; Branch et al. 2002) likely had weak $\mathrm{H} \alpha$ absorption in their early-time spectra from a small residual hydrogen envelope. We will use the catchall term "stripped-envelope SNe" (Clocchiatti 
et al. 1996) to refer to SNe from massive stars lacking the thick hydrogen envelopes of typical SNe II.

The prototypical SN IIb, SN 1993J, occurred in M81 and was one of the brightest $\mathrm{SNe}$ of the twentieth century. Its proximity and late-time brightness at all wavelengths due to strong interaction with circumstellar material (CSM) enabled numerous detailed studies that make it the second-best-studied core-collapse SN after SN 1987A in the Large Magellanic Cloud (Wheeler \& Filippenko 1996). Furthermore, one of the youngest and best-studied SN remnants in the Galaxy, Cassiopeia A (Cas A), was recently revealed to have been an $\mathrm{SN}$ IIb on the basis of spectroscopy of scattered-light echoes from the SN (Krause et al. 2008).

We gained the opportunity to study another nearby strippedenvelope SN in great detail when Mostardi et al. (2008) announced the discovery of a possible SN at an unfiltered magnitude of 16.1 in the nearby $(d \approx 9.6 \mathrm{Mpc}$; Pastorello et al. 2008, hereafter P08) galaxy NGC 4490 on 2008 March 3.45 (UT dates are used throughout this paper). The following night, the new object was spectroscopically confirmed as a peculiar SN II (Blondin et al. 2008). The spectroscopic classification continued to evolve as the SN aged, with Chornock et al. (2008) identifying the incipient $\mathrm{He}$ I lines diagnostic of SNe IIb by March 9.6 and Marion et al. (2008) using near-infrared (NIR) spectra of SN 2008ax on March 14.5 to classify it as typical of $\mathrm{SNe} \mathrm{Ib}$.

Fortuitously, Arbour (2008) had imaged NGC 4490 on March 3.19 and did not detect SN 2008ax to a limiting unfiltered magnitude of 19.5 (P08). The SN must have risen by at least $3.4 \mathrm{mag}$ in the six hours before the first detection by Mostardi et al. (2008), setting a strict limit on the time of shock breakout. Throughout this paper we will assume a time for shock breakout of SN 2008ax of 2008 March 3.32, halfway between the nondetection by Arbour (2008) and the discovery by Mostardi et al. (2008). This unusually early discovery enabled rapid follow-up observations at all wavelengths.

Observations of SNe at very early times are obtained only rarely, but they are important because they reflect the state of the outermost layers of the progenitor star, which are otherwise difficult to study, and provide a crucial link between studies of core-collapse SNe and their progenitors. The ultraviolet (UV) and optical light curves presented by Roming et al. (2009) show the initial phase of rapid cooling of the stellar envelope after shock breakout, a short-lived phenomenon occurring immediately after explosion that has only been observed in a handful of core-collapse SNe. The proximity of the host galaxy enabled Li et al. (2008) and Crockett et al. (2008) to identify a source at the position of SN 2008ax in pre-explosion Hubble Space Telescope images. Unfortunately, the apparent progenitor appears to be blended with other nearby sources, making the interpretation ambiguous. Crockett et al. (2008) found that the progenitor could be modeled as either a massive star that had lost most of its hydrogen envelope in a Wolf-Rayet (W-R) phase or as an interacting binary star.

We conducted an extensive spectroscopic campaign to observe SN 2008ax, starting two days after shock breakout and extending into the nebular phase. Our earliest spectra are sensitive to the composition of the outermost layers of the progenitor, while the latest ones probe the inner core. In addition, five epochs of NIR spectra were taken over the first two months after explosion, starting 10 days after shock breakout. The NIR spectra of stripped-envelope $\mathrm{SNe}$ are less well-studied than those at optical wavelengths, but they provide a different window into the explosion with strong lines from light- and intermediate-mass elements that are blended or obscured at optical wavelengths. The importance of SN 2008ax also led us to obtain three epochs of optical spectropolarimetry. Our first two observations were obtained within nine days of shock breakout and allow us to study the geometry of the outer ejecta, while our final epoch extends our study of the geometry to the early nebular phase.

Optical photometry of SN 2008ax has been presented by P08, who also presented a series of seven optical spectra starting 10 days after shock breakout and a single NIR spectrum obtained after maximum light. Additional photometry and preliminary modeling of the light curve were presented by Tsvetkov et al. (2009). Late-time optical spectra of SN 2008ax were studied in detail by Milisavljevic et al. (2010). Taubenberger et al. (2011) have also presented a large optical and NIR data set. Roming et al. (2009) presented UV and optical light curves, as well as $\mathrm{X}$-ray and radio detections, enabling study of pre-SN mass loss of the progenitor. Roming et al. (2009) also showed two optical spectra taken near maximum light.

In this paper, we add to the previous studies of SN 2008ax by presenting our large and well-sampled spectroscopic data set. The observations are described in Section 2 and the detailed optical-through-NIR spectral evolution is discussed in Section 3. A highlight of our study are the three epochs of optical spectropolarimetry showing large line polarizations beginning shortly after explosion, which are analyzed in Section 4. Implications of these observations are discussed in Section 5.

\section{OBSERVATIONS}

\subsection{Optical Spectroscopy}

We obtained a well-sampled sequence of low-resolution optical spectra of SN 2008ax starting two days after discovery and extending into the early nebular phase. Spectra were obtained using the Kast spectrograph on the Lick Observatory's $3 \mathrm{~m}$ telescope (Miller \& Stone 1993), the Low Resolution Imaging Spectrometer (Oke et al. 1995) on the Keck I 10 m telescope, and the Echellette Spectrograph and Imager (ESI; Sheinis et al. 2002) on the Keck II 10 m telescope. Two-dimensional image reduction and spectral extraction were performed using standard tasks in IRAF. ${ }^{18}$ Flux calibration and removal of telluric absorption features were performed using our own IDL routines (Matheson et al. 2000a). The early-time spectra are shown in Figure 1. A log with the details of all the observations is given in Table 1. Hereafter, we refer to the dates of observation as "day $X$," where $X$ represents the number of days after shock breakout, assumed to be 2008 March 3.32. P08 determined the time of maximum light in the $B$ band to be 19 days later (2008 March $22.2 \pm 0.5)$.

In addition, we obtained high-resolution spectra of SN 2008ax using the High Resolution Echelle Spectrometer (HIRES; Vogt et al. 1994) on Keck I on 2008 March 24 and 25, with exposure times of $1200 \mathrm{~s}$ on each night. Numerous absorption features from the interstellar medium (ISM) of NGC 4490 are present, including diffuse interstellar bands (DIBs; Herbig 1975), which we discuss further in the Appendix. The Na I D1 $\lambda 5890$ and K I $\lambda 7699$ absorption-line profiles are shown in Figure 2. Both lines show absorption from multiple components over the velocity range of $590-670 \mathrm{~km} \mathrm{~s}^{-1}$, so we adopt $630 \mathrm{~km} \mathrm{~s}^{-1}$ as the radial

\footnotetext{
18 IRAF is distributed by the National Optical Astronomy Observatory, which is operated by the Association of Universities for Research in Astronomy, Inc., under cooperative agreement with the National Science Foundation.
} 


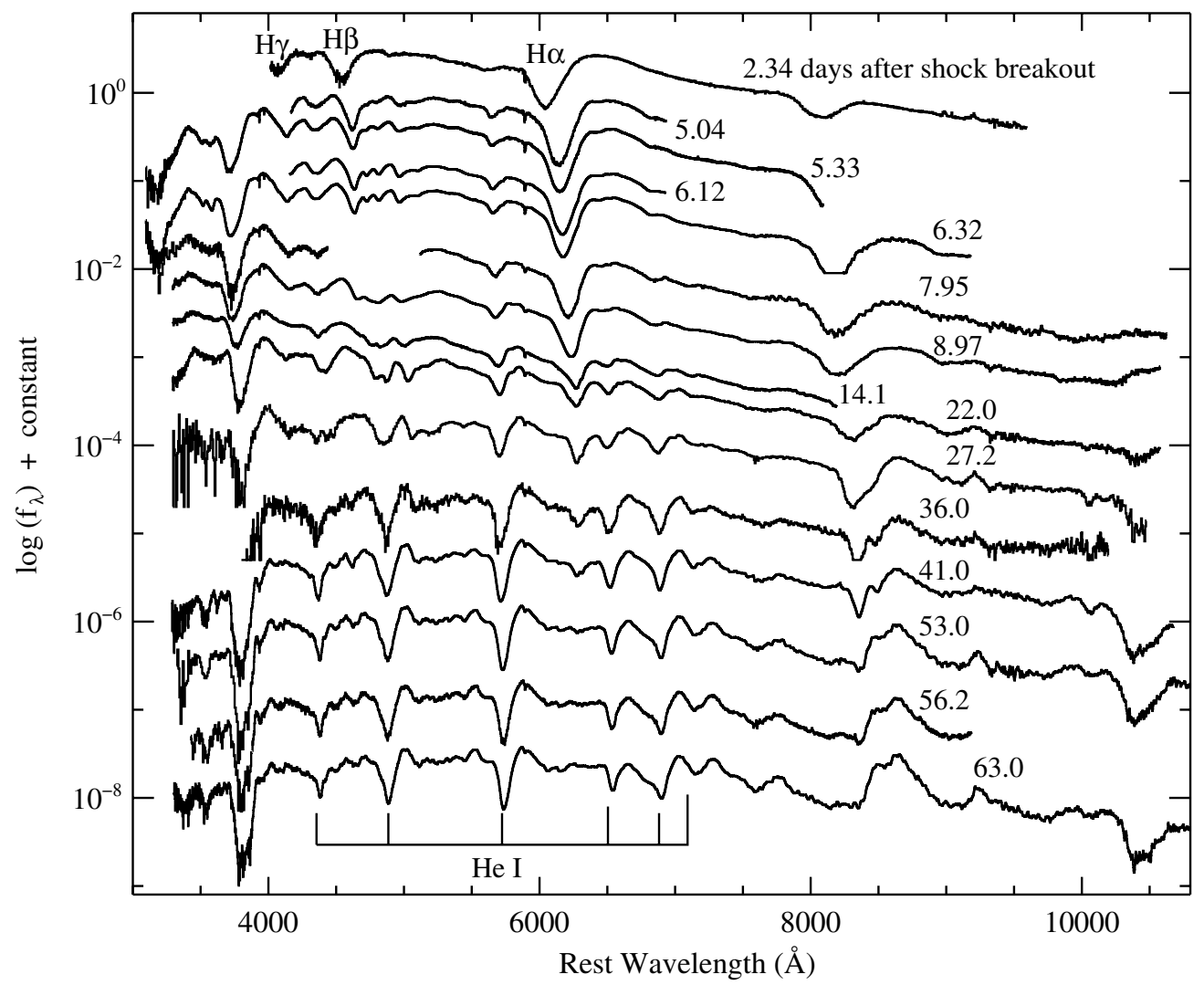

Figure 1. Optical spectral sequence of SN 2008ax during the photospheric phase. Spectra are labeled with their ages in days relative to the assumed shock-breakout date of 2008 March 3.32. Maximum light in the $B$ band was 19 days later (March 22.2; P08). The earliest spectrum shows prominent absorption lines from the hydrogen Balmer series. The He I lines are visible shortly after explosion and gradually come to dominate the spectrum. By two months after explosion, the $\mathrm{H} \alpha$ absorption line disappeared.

Table 1

Log of Low-resolution Optical Spectroscopy

\begin{tabular}{|c|c|c|c|c|c|c|c|}
\hline $\begin{array}{l}\operatorname{Age}^{\mathrm{a}} \\
\text { (days) }\end{array}$ & $\begin{array}{c}\text { UT Date }^{\mathrm{b}} \\
\text { (YYYY-MM-DD.DDD) }\end{array}$ & Instrument $^{\mathrm{c}}$ & $\begin{array}{l}\text { Range } \\
(\AA)\end{array}$ & $\begin{array}{l}\text { Exposure Time } \\
\text { (s) }\end{array}$ & $\begin{array}{l}\text { Seeing } \\
\left({ }^{\prime \prime}\right)\end{array}$ & Airmass & $\begin{array}{c}\text { Clouds? } \\
(\mathrm{y} / \mathrm{n})\end{array}$ \\
\hline 2.34 & $2008-03-05.658$ & ESI & $4000-9600$ & 300 & 0.9 & 1.6 & $\mathrm{n}$ \\
\hline 5.04 & 2008-03-08.360 & Kast & $4170-6950$ & 2700 & 2.3 & 1.0 & $\mathrm{n}$ \\
\hline 5.33 & $2008-03-08.650$ & LRIS & $3100-8110$ & 200 & 0.8 & 1.6 & $\mathrm{n}$ \\
\hline 6.12 & 2008-03-09.437 & $\operatorname{Kast}(\mathrm{p})$ & $4170-6940$ & $4 \times 3000^{d}$ & 2.1 & 1.1 & $\mathrm{n}$ \\
\hline 6.32 & 2008-03-09.638 & LRIS & $3100-9200$ & $560 / 480^{\mathrm{e}}$ & 0.7 & 1.6 & $\mathrm{n}$ \\
\hline 7.96 & $2008-03-11.283$ & Kast & $\begin{array}{l}3110-4450 \\
5130-10650\end{array}$ & 1200 & 2.2 & 1.2 & y \\
\hline 8.97 & $2008-03-12.292$ & $\operatorname{Kast}(\mathrm{p})$ & 4390-9880 & $4 \times 3000^{\mathrm{d}}$ & 2.0 & 1.2 & y \\
\hline 9.07 & $2008-03-12.393$ & Kast & $3300-10600$ & 1200 & 3.0 & 1.0 & y \\
\hline 14.1 & $2008-03-17.423$ & Kast & $3300-8210$ & 600 & 2.3 & 1.0 & $\mathrm{n}$ \\
\hline 22.0 & $2008-03-25.348$ & Kast & $3300-10600$ & 400 & 2.7 & 1.0 & $\mathrm{y}$ \\
\hline 27.2 & 2008-03-30.548 & Kast & $3300-10500$ & 400 & 3.4 & 1.8 & y \\
\hline 36.0 & 2008-04-08.312 & Kast & $3300-10800$ & $600 / 700^{\mathrm{e}}$ & 2.4 & 1.0 & $\mathrm{y}$ \\
\hline 41.0 & 2008-04-13.314 & Kast & $3300-10700$ & 1600 & 2.0 & 1.0 & $\mathrm{y}$ \\
\hline 53.0 & $2008-04-25.351$ & Kast & $3300-10800$ & 1800 & 2.3 & 1.1 & $\mathrm{y}$ \\
\hline 56.2 & $2008-04-28.558$ & LRIS & $3000-9200$ & 500 & 1.9 & 2.3 & $\mathrm{y}$ \\
\hline 63.0 & 2008-05-05.331 & Kast & $3300-10800$ & 1800 & 2.0 & 1.1 & y \\
\hline 85.1 & $2008-05-27.382$ & Kast & $3300-10800$ & $1500 / 1562^{\mathrm{e}}$ & 1.9 & 1.7 & y \\
\hline 99.0 & 2008-06-10.310 & Kast(p) & $4580-10060$ & $4 \times 2400^{\mathrm{d}}$ & 2.5 & 1.6 & $\mathrm{n}$ \\
\hline 118 & 2008-06-29.249 & Kast & $3300-10750$ & 1800 & 2.1 & 1.3 & $\mathrm{y}$ \\
\hline 158 & 2008-08-08.914 & Kast & $3540-10750$ & 2100 & 2.5 & 1.9 & $\mathrm{n}$ \\
\hline
\end{tabular}

Notes.

${ }^{a}$ Age in days relative to the assumed shock-breakout date of 2008 March 3.32.

${ }^{\mathrm{b}}$ Midpoint of observation.

${ }^{\mathrm{c}}$ ESI = Echellette Spectrograph and Imager on the Keck II $10 \mathrm{~m}$ telescope. Kast $=$ Kast spectrograph on the Lick $3 \mathrm{~m}$ telescope; (p) if used in polarimetry mode. LRIS = Low Resolution Imaging Spectrometer on the Keck I 10 m telescope.

${ }^{\mathrm{d}}$ Exposure time was (for example) $3000 \mathrm{~s}$ at each of the four wave plate rotation angles, for a total of 12,000 s.

e Total exposure times on blue/red sides of the spectrograph. 


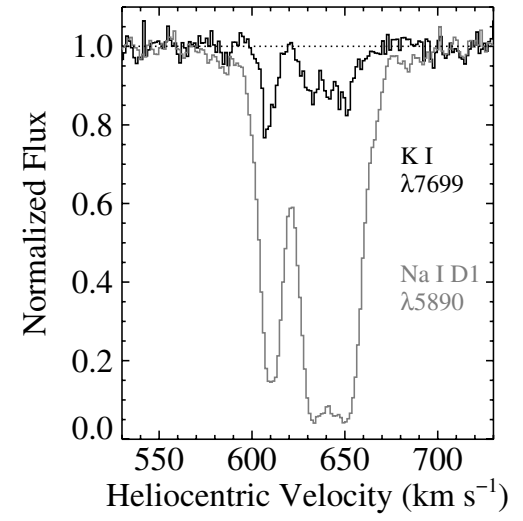

Figure 2. $\mathrm{Na}$ I and $\mathrm{K}_{\mathrm{I}}$ lines from the interstellar medium of NGC 4490 in the HIRES spectrum of SN 2008ax. The Na I absorption is clearly saturated and is not a reliable tracer of the total column along the line of sight to SN 2008ax. We adopt $630 \mathrm{~km} \mathrm{~s}^{-1}$ as the radial velocity of SN 2008ax.

velocity of SN 2008ax. This redshift has been removed from all other spectra in the plots in this paper.

These lines can also be used to estimate the host-galaxy contribution to the reddening of SN 2008ax, which dominates over the Galactic component $(E(B-V)=0.02$ mag; Schlegel et al. 1998). However, the Na I lines, which are usually used to estimate the reddening in SN studies, have multiple saturated components along this line of sight and therefore do not provide a reliable reddening estimate, so instead we use the $\mathrm{K}_{\mathrm{I}} \lambda 7699$ line. The total equivalent width (EW) of the $\mathrm{K}_{\mathrm{I}} \lambda 7699$ absorption over the velocity range of $590-670 \mathrm{~km} \mathrm{~s}^{-1}$ is $0.142 \pm 0.006 \AA$, which corresponds to a predicted reddening of $E(B-V)=$ 0.54 mag using the relationship of Munari \& Zwitter (1997). This value, combined with a detailed comparison of the colors of SN 2008ax to those of other core-collapse SNe (W. Li et al. 2011 , in preparation), leads us to adopt a value for the reddening of the SN of $E(B-V)=0.5$ mag. All spectra in this paper have been corrected for this value of the reddening (assuming that $\left.R_{V}=A_{V} / E(B-V)=3.1\right)$. This value is higher than that used by $\mathrm{P} 08(E(B-V)=0.3 \mathrm{mag})$ and Crockett et al. (2008), which was estimated using low-resolution spectra of the saturated $\mathrm{Na}$ I line. Our spectroscopic analysis is insensitive to the exact value of the adopted reddening.

\subsection{NIR Spectroscopy}

We observed SN 2008ax on five epochs (2008 March 13.51, 14.52, 25.44, April 12.48, and May 8.30; days 10, 11, 22, 40, and 66) using the SpeX instrument on the $3 \mathrm{~m} \mathrm{NASA}$ Infrared Telescope Facility (Rayner et al. 2003). Our first observation was taken in cross-dispersed mode (SXD; $R=$ $\lambda / \Delta \lambda \approx 1200$ ), but the remaining observations used the 0 .5 slit in low-resolution prism mode (LRS; $R \approx 200$ ) to cover the wavelength range $0.7-2.4 \mu \mathrm{m}$. The reductions were performed using the Spextool package (Cushing et al. 2004) and corrections for telluric absorption were made following the method of Vacca et al. (2003). A thorough discussion of the data acquisition and reduction process in the context of SN observations was presented by Marion et al. (2009). The NIR spectra of SN 2008ax are shown in Figure 3.

\subsection{Spectropolarimetry}

We obtained three epochs of optical spectropolarimetry using Kast at the Lick $3 \mathrm{~m}$ telescope, twice at early phases (days 6 and 9) and once in the early nebular phase (day 99). The data were

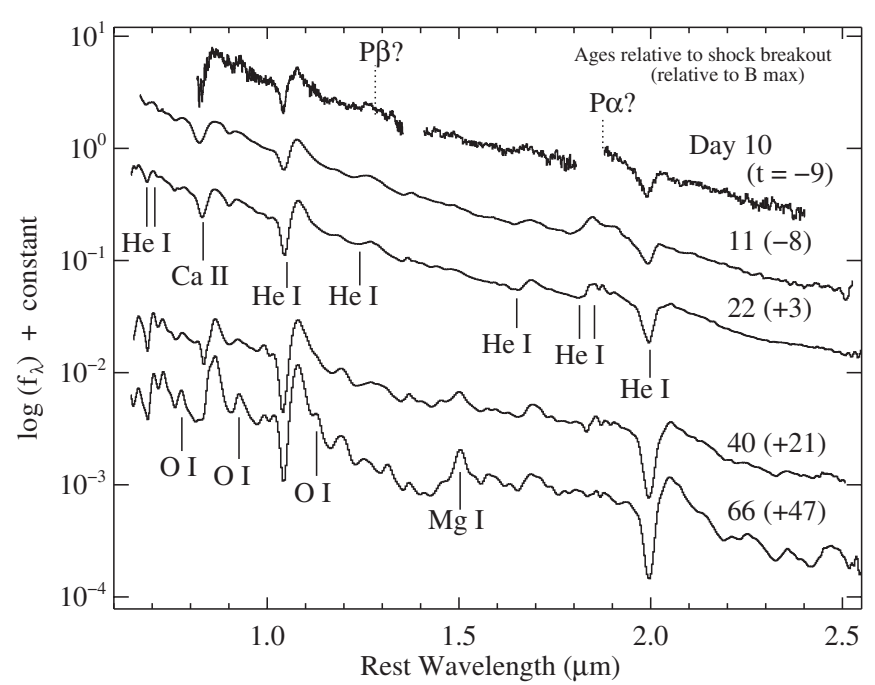

Figure 3. NIR spectra of SN 2008ax. The spectra are labeled by dates relative to shock breakout and relative to maximum light in $B$ (in parentheses). The spectra obtained prior to maximum light are smooth, with few strong lines. Dotted lines mark the rest wavelengths of $\mathrm{P} \alpha$ and $\mathrm{P} \beta$, the strongest expected lines of hydrogen, but there are no clear associated emission peaks. The expected locations of absorption minima from $\mathrm{He}$ I lines at $0.7065,0.7281,1.0830$, $1.2787,1.7002,1.8689,1.9089$, and $2.0581 \mu \mathrm{m}$, blueshifted by $9000 \mathrm{~km} \mathrm{~s}^{-1}$, are labeled in the near-maximum-light spectrum (day 22). After maximum light, blends of features from neutral and singly ionized intermediate-mass elements become more prominent.

reduced in a standard manner, following the method described by Miller et al. (1988) and implemented by Leonard et al. (2001). All of the spectropolarimetry was taken using only the red side of Kast. On days 9 and 99 we used a GG455 order-blocking filter, but our spectral range extended to wavelengths greater than $9000 \AA$, where some second-order light is likely present. Our analysis does not rely on data at these wavelengths, although the red continuum of SN 2008ax due to its high extinction implies that contamination should be small.

We observed polarimetric standards from Schmidt et al. (1992) to set the zero point of the half-wave plate retarder and low-polarization standards from the literature (Schmidt et al. 1992) to verify that instrumental polarization was negligible $(P<0.1 \%)$. On day 99 , an instrumental failure unrelated to the polarimeter unit prevented us from obtaining polarization standards, so we used the angle-correction curve from day 9. Past experience has shown that the zero point of the Kast wave plate is usually stable, with only small drifts over time. The day 6 and 9 zero points agree to within $1^{\circ}$ of data taken as far in the past as at least 2007 May 10 (Chornock et al. 2010). Our next available spectropolarimetry data after the day 99 data set were taken on 2008 December 31 and the zero point had shifted by 2.7 relative to day 9 . Therefore, we believe our lack of observations of standards on day 99 results in little uncertainty in our calibrations.

\section{SPECTRAL EVOLUTION}

The optical spectral evolution of SN 2008ax in the photospheric phase can be seen in Figure 1. The sequence shows an obvious trend as the hydrogen Balmer lines decrease in strength and shift to lower velocities over time while the He I lines strengthen and dominate the spectra after maximum light (see also P08). 


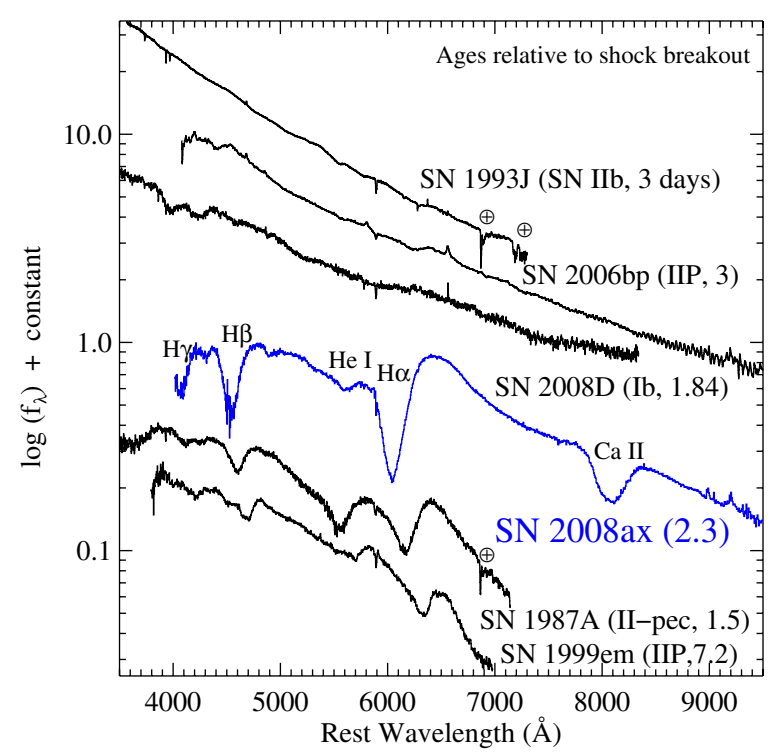

Figure 4. Very early spectra of several core-collapse SNe of a variety of spectral types compared to SN 2008ax. The spectra are labeled with their ages relative to assumed times of shock breakout. The moderate-resolution ESI spectrum of SN 2008ax has been rebinned for display purposes. Spectra of other objects were collected from the literature (SN 1987A: Menzies et al. 1987; SN 1993J: Filippenko et al. 1993; SN 1999em: Leonard et al. 2002; SN 2006bp: Quimby et al. 2007; SN 2008D: Modjaz et al. 2009). Uncorrected telluric absorptions in the literature spectra are marked with " $\oplus$ ". All spectra in this and subsequent comparison plots were corrected for the reddening values listed in Table 2, assuming that $R_{V}=3.1$.

(A color version of this figure is available in the online journal.)

Table 2

Adopted Reddenings for Comparison SN Spectra

\begin{tabular}{lll}
\hline \hline Object & $\begin{array}{c}E(B-V) \\
(\mathrm{mag})\end{array}$ & \multicolumn{1}{c}{ Note about Source } \\
\hline SN 1987A & 0.17 & Walker \& Suntzeff (1990) \\
SN 1987K & 0.5 & To match continuum shape with SN 2008ax \\
SN 1990B & 0.85 & Clocchiatti et al. (2001) \\
SN 1993J & 0.3 & "Moderate" value from Richmond et al. (1994) \\
SN 1999em & 0.1 & Baron et al. (2000) \\
SN 1999ex & 0.5 & To match observed colors with SN 2008ax \\
SN 2000H & 0.16 & Benetti et al. (2000) \\
SN 2005bf & 0.045 & Folatelli et al. (2006) \\
SN 2006bp & 0.4 & Dessart et al. (2008) \\
SN 2008D & 0.6 & Modjaz et al. (2009) \\
\hline
\end{tabular}

\subsection{Day 2 Spectrum}

Our first spectrum, taken just 2.34 days after shock breakout, is one of the earliest spectra of an SN ever taken. We compare it in Figure 4 to the earliest spectra of other core-collapse $\mathrm{SNe}$ of various spectral types. Throughout this paper, we correct the reference $\mathrm{SN}$ spectra for reddening to facilitate comparison, using the adopted values listed in Table 2. In a few cases, good reddening estimates do not exist, so we do not list those objects in Table 2 or correct their spectra for reddening.

SN spectral types are defined using optical spectra near maximum light (e.g., Filippenko 1997), but at early times the spectra do not correspond in a simple manner to classes based on their appearances at later times. The diversity of these spectra is related to the state of the progenitor star at the time of explosion and to the presence or absence of CSM. The SN IIb 1993J and the SN IIP 2006bp both have blue spectra with relatively weak spectral features. Both also showed transient narrow emission features from the CSM that was overrun by the SN ejecta within a few days (Benetti et al. 1994; Garnavich \& Ann 1994; Quimby et al. 2007). The SNe IIP 1999em and 2006bp resembled each other at later epochs, but in these early-time spectra the Balmer lines clearly have different strengths. Similarly, the early spectra of SNe 2008D (Soderberg et al. 2008; Modjaz et al. 2009; Mazzali et al. 2008; Malesani et al. 2009) and 2008ax are very different, with SN 2008ax showing strong Balmer lines from its very thin outer $\mathrm{H}$ envelope, but by maximum light the two $\mathrm{SNe}$ showed a close resemblance (see below).

The most distinctive aspect of SN 2008ax in Figure 4 is the series of extremely strong absorptions from Balmer transitions and the Ca II NIR triplet with very high expansion velocities. The absorptions are clearly deeper than in any of the other objects. By comparison, SNe 1993J and 2008D show only weak features. Modjaz et al. (2009) attributed the strongest features in this SN 2008D spectrum to $\mathrm{C}_{\text {III }}$ and N III, two species indicative of higher temperatures than the low-ionization $\mathrm{H}$ and $\mathrm{Ca}$ II lines present in the spectrum of SN 2008ax. The weak features in the earliest spectra of SN 1993J are not well understood (Baron et al. 1995), but $\mathrm{H} \alpha$ is likely present.

The large velocities of the Balmer lines in our day 2.34 SN 2008ax spectrum, even in comparison to SN 1987A, are also evident in Figure 4. The flux minimum of $\mathrm{H} \alpha$ is blueshifted by $24,600 \mathrm{~km} \mathrm{~s}^{-1}$ and the blue wing of absorption extends outward to greater than $33,000 \mathrm{~km} \mathrm{~s}^{-1}$, with the precise value slightly uncertain due to the difficulty in defining a continuum with such broad lines. Blondin et al. (2008) reported that on the previous night (day 1.05 after shock breakout) the minimum of $\mathrm{H} \alpha$ was blueshifted by $26,600 \mathrm{~km} \mathrm{~s}^{-1}$. Similarly, the CaII NIR triplet absorption minimum was near $8075 \AA$, with the blue wing extending outward to $7800 \AA$ (blueshifts of 18,100 and $28,500 \mathrm{~km} \mathrm{~s}^{-1}$ relative to the $g f$-weighted line centroid of $8579 \AA$, respectively). The $\mathrm{H} \alpha$ profile in the earliest SN 1987A spectrum from Menzies et al. (1987), taken 1.5 days after explosion (and shown in Figure 4), has a flux minimum blueshifted by $18,300 \mathrm{~km} \mathrm{~s}^{-1}$. By comparison, the $\mathrm{H} \alpha$ minimum of the normal SN IIP 2006bp was blueshifted by only $15,400 \mathrm{~km} \mathrm{~s}^{-1}$ three days after shock breakout (Quimby et al. 2007). ${ }^{19}$ The broad-lined SN IIb 2003bg did have $\mathrm{H} \alpha$ absorption velocities near $20,000 \mathrm{~km} \mathrm{~s}^{-1}$ in the earliest spectra (Hamuy et al. 2009; Mazzali et al. 2009).

Matzner \& McKee (1999) investigated the effects of SN shock waves on the outer envelopes of their progenitors and produced expressions for the maximum ejecta velocities. For typical corecollapse SN parameters (e.g., $M_{\text {ejecta }}=10 M_{\odot}, E=10^{51} \mathrm{erg}$ ), they find that the outer layers of SN progenitors with extended convective envelopes, such as the red supergiant progenitors of SNe IIP (e.g., Smartt et al. 2009), are only accelerated up to velocities of $\sim 13,000 \mathrm{~km} \mathrm{~s}^{-1}$. The outer layers of compact progenitors with radiative envelopes, such as blue supergiants or helium stars, can be accelerated up to $\sim 33,000 \mathrm{~km} \mathrm{~s}^{-1}$ with the same SN parameters. The very high ejecta velocities of SN 2008ax are evidence of a compact progenitor star that is independent of the direct analysis of pre-explosion photometry of the progenitor (Crockett et al. 2008) or the early-time light curve (Roming et al. 2009).

If we adopt the derived values for the explosion energy and mass of the ejecta $\left(M_{\text {ejecta }}=2.9 M_{\odot}, E=0.5 \times 10^{51} \mathrm{erg}\right)$ from the analysis of Roming et al. (2009) and keep the fiducial

\footnotetext{
19 We have adopted a date for the shock breakout of SN 2006bp of 2006 April 7.9, following the model fits of Dessart et al. (2008), which is a day earlier than assumed by Quimby et al. (2007).
} 
values for the other parameters, the relationships of Matzner \& McKee (1999) predict that the highest expansion velocities for SN 2008ax should be $\sim 37,000 \mathrm{~km} \mathrm{~s}^{-1}$, consistent with the optical observations. However, Martí-Vidal et al. (2009) used Very Long Baseline Interferometry at $23 \mathrm{GHz}$ to measure an expansion velocity for the $\mathrm{SN}$ shock of $(5.2 \pm 1.3) \times 10^{4} \mathrm{~km} \mathrm{~s}^{-1}$, indicating that our optical observations are (unsurprisingly) not sensitive to the very highest velocity material. If instead we had adopted the explosion parameters from the lightcurve modeling of Tsvetkov et al. (2009) $\left(M_{\text {ejecta }}=3.8 M_{\odot}\right.$, $\left.E=1.5 \times 10^{51} \mathrm{erg}, R_{*}=600 R_{\odot}\right)$, we would get a maximum velocity of $28,300 \mathrm{~km} \mathrm{~s}^{-1}$. This value is clearly an underestimate due to the overly large progenitor radius, which also results in their model overpredicting the observed luminosity of the envelope cooling emission in the early light curve.

As mentioned above, both the SN IIb 1993J and the SN IIP 2006bp exhibited narrow emission lines in their earliest spectra indicative of CSM ionized by both the SN and the CSM interaction shock. SN 2006bp had both the expected narrow Balmer lines as well as emission from higher-ionization material represented by He II $\lambda \lambda 4200,4686$, and C IV $\lambda 5805$ (Quimby et al. 2007). The stronger CSM interaction component of SN 1993J created high-temperature gas in the shock, which in turn resulted in sufficient ionization in the unshocked CSM to produce emission from [Fex] $\lambda 6375$ and [Fe XIV] $\lambda 5303$ (Benetti et al. 1994; Garnavich \& Ann 1994).

Our moderate-resolution ESI spectrum from day 2.34 afforded an excellent opportunity to search for such features in SN 2008ax. However, none are detected. We used the noise level in the spectrum to set $3 \sigma$ limits on the EWs of any unresolved narrow emission lines of $0.05-0.15 \AA$, dependent on wavelength. These limits are significantly lower than the observed EWs of the features in SN 2006bp (0.7-3.1 $\AA$; Quimby et al. 2007) or SN 1993J (0.11-0.3 A; Benetti et al. 1994) at similar epochs. When scaled to the photometry provided by P08, the upper limits in flux at $(\lambda 4686, \lambda 6375)$ are $(1.5,0.8) \times 10^{-16} \mathrm{erg} \mathrm{cm}^{-2} \mathrm{~s}^{-1}$, which correspond to dereddened luminosities of $\lesssim(9.5,3.1) \times$ $10^{36} \mathrm{erg} \mathrm{s}^{-1}$ at a distance of 9.6 Mpc (P08).

Assuming that the narrow-line fluxes are powered by recombination, the upper limit on the narrow $\mathrm{H} \alpha$ flux sets a limit on the mass-loss rate of the progenitor (e.g., Chugai \& Danziger 2003). Using the measurement of the shock velocity from the radio data of $52,000 \mathrm{~km} \mathrm{~s}^{-1}$ (Martí-Vidal et al. 2009), we obtain a limit of $\dot{M} \lesssim 10^{-5} M_{\odot} \mathrm{yr}^{-1}\left(v_{w} / 10 \mathrm{~km} \mathrm{~s}^{-1}\right)\left(0.5 / X_{\mathrm{H}}\right)$, where $v_{w}$ is the velocity of progenitor's wind and $X_{\mathrm{H}}$ is the hydrogen mass fraction of the wind material, which is likely to be lower than the solar value due to the stripped nature of the progenitor. This compares well with the mass-loss rate directly estimated from the X-ray emission of SN 2008ax by Roming et al. (2009) of $\dot{M}=(9 \pm 3) \times 10^{-6} M_{\odot} \mathrm{yr}^{-1}\left(v_{w} / 10 \mathrm{~km} \mathrm{~s}^{-1}\right)$, though they assumed that the velocity of the SN shock was only $10,000 \mathrm{~km} \mathrm{~s}^{-1}$. This is roughly an order of magnitude lower than the mass-loss rate of SN 1993J (Roming et al. 2009) and is consistent with the lack of other signatures of CSM interaction in the spectra of SN 2008ax.

\subsection{Before Maximum Light}

The optical spectra of SN 2008ax evolved rapidly over the next 17 days on the rise to maximum light, but were typical for a SN IIb in the early hydrogen-dominated phase (see also P08). A comparison to the prototypical SNe IIb 1987K (Filippenko 1988) and 1993J (Nomoto et al. 1993; Schmidt et al. 1993; Filippenko et al. 1993; Swartz et al. 1993) at early times is shown

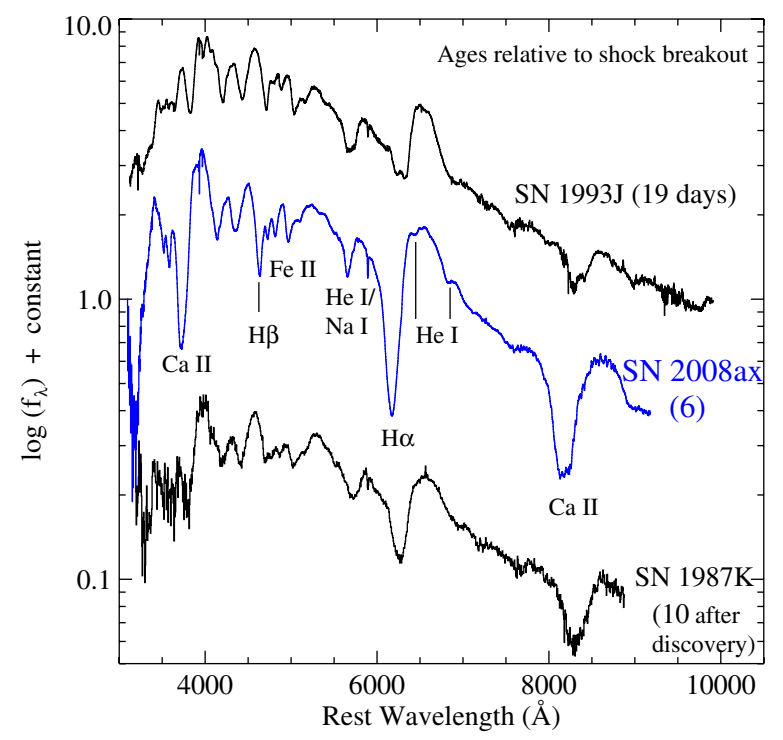

Figure 5. Spectra of $\mathrm{SNe} \mathrm{IIb}$ in the hydrogen-dominated phase. Important spectral features are labeled. The SN 1993J spectrum is from 1993 April 15 (Filippenko et al. 1993) and a shock-breakout date of 1993 March 27.5 has been assumed (Lewis et al. 1994). The spectrum of SN 1987K is from 1987 August 7 (Filippenko 1988) and the listed age is relative to the discovery on 1987 July 28 because the epoch of explosion is not tightly constrained.

(A color version of this figure is available in the online journal.)

in Figure 5. The spectra continue to be dominated by strong Balmer absorption lines, as well as the usual low-ionization species typical of SNe II (e.g., Ca II and Fe II). Na I D also likely contributes to the absorption due to He i $\lambda 5876$.

In addition, notches have appeared in the $\mathrm{H} \alpha$ profile due to He I $\lambda 6678$ and $\lambda 7065$. The day 2.34 spectrum did not show either line, but they developed sometime between day 2 and day 5 (Figure 1). The day 2 spectrum did exhibit He I $\lambda 5876$ (Figure 4), which was even stronger the previous night (Blondin et al. 2008). The $\lambda 5876$ line in the earliest spectra of SN 2008ax was likely to be thermally excited, as is typical in very young SNe II (cf. the other SNe II in Figure 4). Thermal excitation would also explain why the strength of $\lambda 5876$ decreased from day 1 (Blondin et al. 2008) to day 2 as the photospheric temperature cooled. This effect is also seen in normal SNe IIP, whose optical He I lines disappear after the photospheric temperature drops below $\sim 10,000 \mathrm{~K}$ (e.g., Leonard et al. 2002). The optical helium lines at later times in $\mathrm{SNe} \mathrm{Ib}$ and IIb are excited by nonthermal electrons created by exposure to ${ }^{56} \mathrm{Ni}$ (Lucy 1991; Swartz et al. 1993).

The appearance of He I $\lambda 6678$ and $\lambda 7065$ in the first week after explosion and their increasing strength thereafter was a sign that the progenitor of SN 2008ax did not have the thick hydrogen envelope of typical SNe II and instead was partially stripped. SNe which exhibit strong hydrogen lines at early times like SNe II but which transition to the helium-dominated spectra of $\mathrm{SNe} \mathrm{Ib}$ at late times are known as $\mathrm{SNe} \mathrm{IIb}$, a possibility that was first predicted theoretically by Woosley et al. (1987). The most well-studied example of an object undergoing such a transition was SN 1993J (see Matheson et al. 2000b and references therein). SN 2008ax was more stripped than SN 1993J and came to resemble a SN Ib even sooner after explosion (P08).

The premaximum NIR spectra of SN 2008ax (Figure 3) are remarkably smooth and devoid of prominent spectral features. The rest wavelengths of $\mathrm{P} \alpha$ and $\mathrm{P} \beta$ are marked with dotted lines and question marks in Figure 3. $\mathrm{P} \alpha$ falls in a region of 


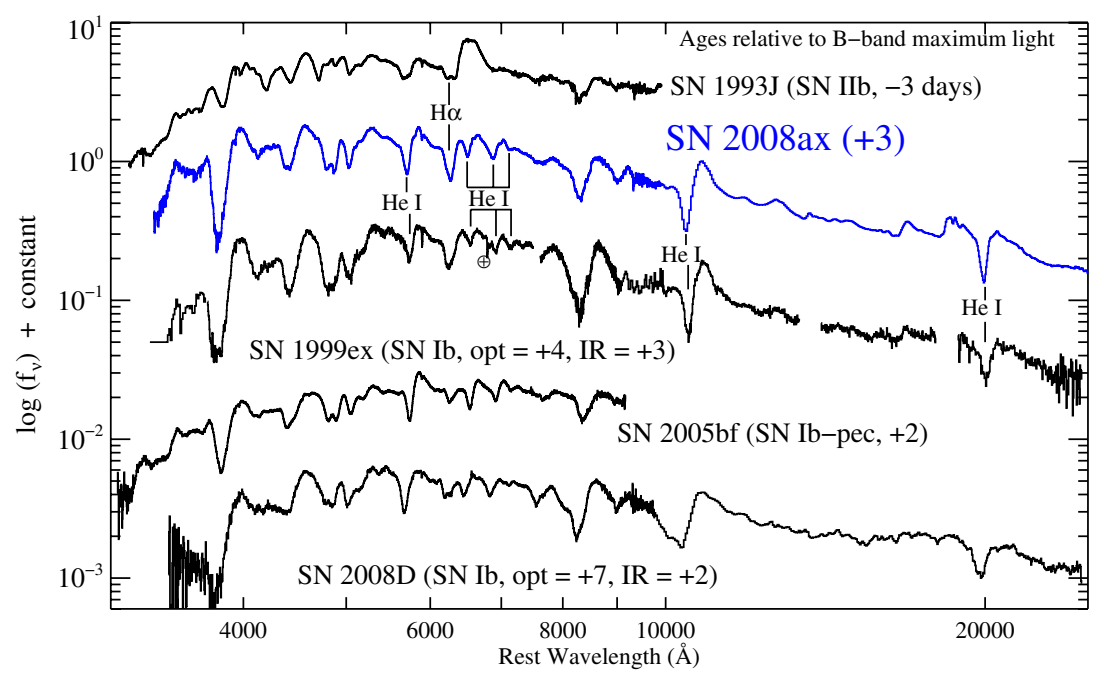

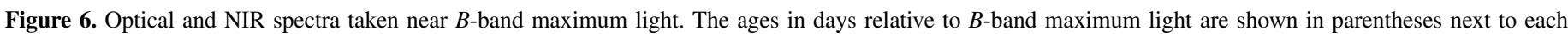

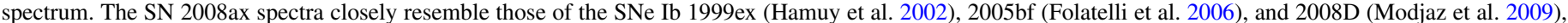

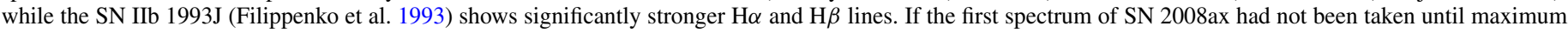

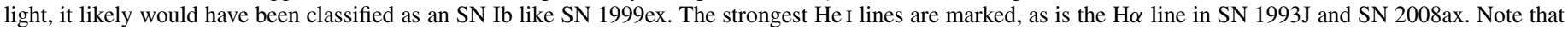
SN 1999 ex likely also has H $\alpha$ absorption. A weak telluric absorption in the SN 1999 ex spectrum is marked with " $\oplus$ ".

(A color version of this figure is available in the online journal.)

strong telluric absorption between the $H$ and $K$ bands, but there is not a prominent P-Cygni emission feature centered at that wavelength. At best, $\mathrm{P} \beta$ contributes to a subtle wiggle in the spectrum, but He I $1.279 \mu \mathrm{m}$ is close in wavelength and likely contributes to that feature. P08 attributed this feature solely to $\mathrm{P} \beta$ in their single NIR spectrum, taken on day 26 (7 days after $B$-band maximum light). $\mathrm{Br} \gamma$ at $2.166 \mu \mathrm{m}$ also does not appear to be present. Collectively, evidence for hydrogen is weak or lacking relative to the contemporaneous optical spectra. Another piece of evidence against significant Paschen absorption is that the features near $\mathrm{P} \alpha$ and $\mathrm{P} \beta$ did not weaken substantially as the $\mathrm{SN}$ aged, unlike the optical Balmer lines. Instead, the strongest features in the 1-2.5 $\mu \mathrm{m}$ spectral range are absorptions from He I 1.083 and $2.058 \mu \mathrm{m}$. In addition to those prominent features, the expected locations of absorptions from other NIR He I lines are marked in Figure 3 and they appear to match several of the weaker features.

The NIR spectra of SN 1993J were quite different. While at the earliest times the NIR spectra were very smooth and featureless (similar to the optical spectra; Figure 4), weak $\mathrm{P} \beta$ became apparent by about four days after shock breakout (Wheeler et al. 1993; Matthews et al. 2002). P $\beta$ grew in prominence on the rise to maximum light ${ }^{20}$ and beyond, peaking in strength 30-40 days after explosion (Matthews et al. 2002). The available NIR spectra of SN 1993J are limited in wavelength coverage and spectral resolution, so the appearance of the NIR helium lines is more difficult to characterize, although He I 1.083 and $2.058 \mu \mathrm{m}$ were definitely strong by 37 days after explosion (Swartz et al. 1993; Matthews et al. 2002). The lack of strong P $\beta$ in our SN 2008ax spectra taken as soon as 10 days after shock breakout, and the relative prominence of the helium lines being quite different from SN 1993J at similar epochs, led Marion et al. (2008) to classify SN 2008ax as an SN Ib.

\footnotetext{
20 In this work we use the term "maximum light" to refer to the second peaks of the SNe 1993J and 2005bf light curves. At least in the case of SN 1993J, it is clear that the second peak is physically analogous to maximum light in the other objects (e.g., Nomoto et al. 1993; Podsiadlowski et al. 1993).
}

\subsection{Early Post-maximum Phase}

\subsubsection{Spectral Comparisons}

The optical and NIR spectra of SN 2008ax near maximum light are plotted in Figure 6 along with those of the $\mathrm{SNe} \mathrm{Ib}$ 1999ex (Hamuy et al. 2002) and 2008D (Soderberg et al. 2008; Modjaz et al. 2009; Mazzali et al. 2008; Malesani et al. 2009), as well as optical spectra of the peculiar SN Ib/c 2005bf (Folatelli et al. 2006) and the SN IIb 1993J (Filippenko et al. 1993). SN 1993J stands out as the most distinct from the others because of its prominent $\mathrm{H} \alpha$ emission. SN 2008ax and SN 1999ex are very similar over the full optical-through-NIR spectral range, with the usual SN Ib P-Cygni features of He I, Fe II, Ca II, and O I all being present. SN 2008ax also more closely resembles the SN Ib 2008D than SN 1993J at this epoch.

The largest difference between SN 2008ax and SN 2008D at this time is in the absorption near $1.05 \mu \mathrm{m}$, which is clearly broader in SN 2008D. As discussed by Taubenberger et al. (2006) and Modjaz et al. (2009), SNe Ib/c commonly exhibit an absorption near $1.04 \mu \mathrm{m}$ that is likely a blend of several species, with potential contributions from $\mathrm{Mg}$ II, Fe II, C I, Ca II, S I, and $\mathrm{Si}$ I, while only $\mathrm{SNe} \mathrm{Ib}$ have strong absorptions from both of the $\mathrm{He}$ I lines at rest wavelengths of 1.083 and $2.058 \mu \mathrm{m}$. The absorption near $1.05 \mu \mathrm{m}$ in SN 2008D had a multi-component structure that was likely a blend of both the usual $1.04 \mu \mathrm{m}$ absorption blend and He I $1.083 \mu \mathrm{m}$. SNe 1999ex and 2008ax appear to have absorption from only the He I line, as the profile of the $1.05 \mu \mathrm{m}$ absorption is similar in velocity space (relative to $1.083 \mu \mathrm{m}$ ) to that of the He I $2.058 \mu \mathrm{m}$ line, but with the flux minimum being at a slightly higher velocity. Both objects appear to lack the additional component near $1.04 \mu \mathrm{m}$ that results in the broader appearance of the absorption in SN 2008D.

The similarity between the spectra of SNe 2008D and 2008ax near maximum light is in contrast to their remarkably different spectra shortly after explosion (Figure 4). Unfortunately, no comparably early spectra of SN 1999ex exist. That comparison would have been especially interesting because the early-time light curve of SN 2008ax during the envelope-cooling phase 
immediately following shock breakout was more similar to that of SN 1999ex (Stritzinger et al. 2002; Roming et al. 2009). The SN Ib 2007Y also had an early-time light curve which closely resembled SN 1999ex, and that object did show strong high-velocity $\mathrm{H} \alpha$ absorption in the earliest spectrum, taken 14 days before maximum light (Stritzinger et al. 2009). Despite the strength of the Balmer lines in the SN 2008ax spectra shortly after explosion, the strongest evidence for hydrogen after maximum light is the absorption due to $\mathrm{H} \alpha$ near $6270 \AA$.

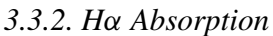

A robust detection of $\mathrm{H} \alpha$ absorption is an important insight from our study of SN 2008ax. Many SNe Ib have an absorption of variable depth near $6300 \AA$ for which several identifications have been proposed. For example, SN 1999ex clearly shows such a feature in Figure 6, similar to the one seen in SN 2008ax. The original study of Hamuy et al. (2002) identified it as Si II $\lambda 6355$, but Elmhamdi et al. (2006) and Parrent et al. (2007) argued that it was due to $\mathrm{H} \alpha$. Branch et al. (2002) analyzed a large fraction of available SN Ib spectra and found that the $6300 \AA$ absorption was present in most of the objects in their sample. They examined several possibilities for the identification of the line and found that $\mathrm{H} \alpha$ was the most natural fit. Our densely sampled spectral sequence of SN 2008ax in Figure 1 shows that the strong, obvious $\mathrm{H} \alpha$ line at the earliest times smoothly evolved into the $6270 \AA$ absorption seen after maximum light before disappearing by $\sim 50$ days after explosion.

We have plotted a comparison of the post-maximum-light spectra of several of the hydrogen-rich $\mathrm{SNe} \mathrm{Ib}$ and $\mathrm{SNe} \mathrm{IIb}$ from Branch et al. (2002) in Figure 7. The $\mathrm{H} \alpha$ absorption of SN 2008ax is not notably strong relative to the other objects, in contrast with the strength of Balmer lines in SN 2008ax relative to other core-collapse $\mathrm{SNe}$ in the earliest spectra (Figure 4). Most SNe are not observed as soon after explosion as SN 2008ax. In many cases, the first spectrum of an $\mathrm{SN}$ is taken around or shortly after maximum light. If the first spectrum of SN 2008ax had not been taken until after maximum light, it probably would have been classified as an SN Ib like SN 1999ex. Similarly, the time evolution of the radio emission from CSM interaction in $\mathrm{SN} 2008 \mathrm{ax}$ is more typical of $\mathrm{SNe} \mathrm{Ib} / \mathrm{c}$ than the strong radio emission seen in SN 1993J (Roming et al. 2009).

We used the SuperNova IDentification program (SNID; Blondin \& Tonry 2007) to cross-correlate the near-maximumlight (day 22) optical spectrum of SN 2008ax with the default library of SN templates that are distributed with SNID. The best matches were all with SNe 2005bf (Tominaga et al. 2005; Folatelli et al. 2006) and 1999ex (Hamuy et al. 2002) near maximum light. SNID also finds matches for the day 14 and 27 spectra with SNe IIb such as SN 1996cb (Qiu et al. 1999) and SN 2000H (Branch et al. 2002) or the hydrogen-rich SNe Ib 1999dn and 1999di (Matheson et al. 2001). By day 41 (22 days after $B$ maximum), the best SNID matches are many of the same $\mathrm{SNe} \mathrm{Ib}$ as shown in Figure 7. These SNID-based comparisons (using the default set of templates) are probably representative of the likely spectroscopic classification of SN 2008ax in the International Astronomical Union Circulars had it not been discovered so soon after explosion.

This suggests that a substantial fraction of objects classified as $\mathrm{SNe} \mathrm{Ib}$ might have similar amounts of hydrogen in their outer ejecta as SN 2008ax. Another example is the SN Ib 2007Y (Stritzinger et al. 2009), whose earliest spectra show strong high-velocity $\mathrm{H} \alpha$ absorption, but by maximum light also closely

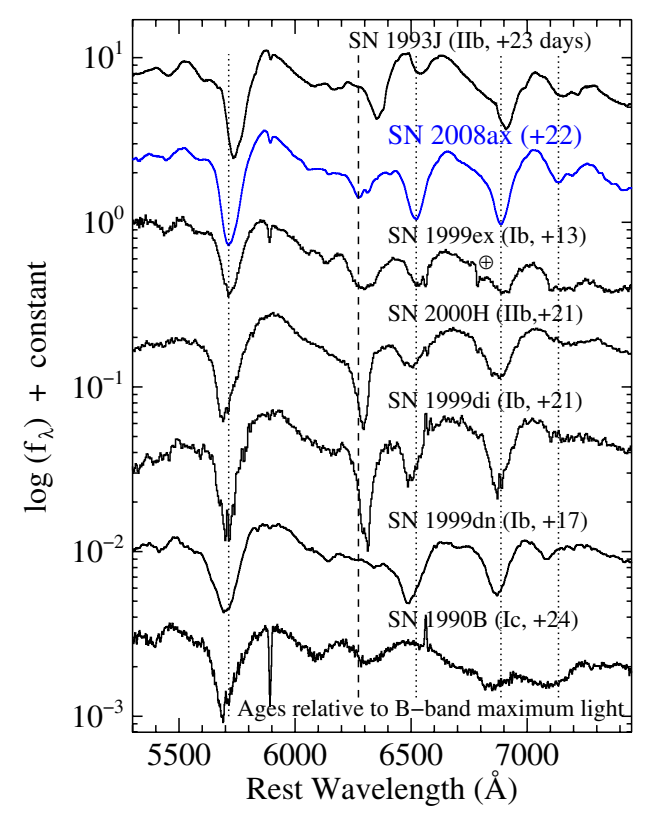

Figure 7. Comparison of SN 2008ax to spectra of several stripped-envelope SNe a few weeks after maximum light. The vertical dashed line marks the location of $\mathrm{H} \alpha$ in the spectrum of SN 2008ax and the vertical dotted lines mark the locations of the He I $\lambda \lambda 5876,6678,7065$, and 7281 absorption minima in SN 2008ax. The minima correspond to slightly different velocities depending on the optical depth in each line (see Table 3). The comparison spectra come from two Type IIb SNe (SN 1993J: Filippenko et al. 1994; SN 2000H: Branch et al. 2002), two "hydrogen-rich" SNe Ib (SN 1999di: Matheson et al. 2001; SN 1999ex: Hamuy et al. 2002), one normal SN Ib (SN 1999dn, which did have $\mathrm{H} \alpha$ absorption at earlier times: Matheson et al. 2001), and a normal SN Ic (SN 1990B: Clocchiatti et al. 2001). A telluric absorption in the SN 1999ex spectrum is marked with " $\oplus$ ".

(A color version of this figure is available in the online journal.)

resembled SN 2005bf. An analysis of the nebular spectra by Maurer et al. (2010a) concluded that SN 2007Y and SN 2008ax probably ejected similar amounts of hydrogen. As discussed by Branch et al. (2002), this has important implications for the nature of their progenitors. Additionally, the division between spectroscopic classifications of objects as $\mathrm{SNe} \mathrm{Ib}$ versus IIb may in some cases depend on the timing of the initial spectroscopy, although not for those $\mathrm{SNe}$ whose progenitors retain as much hydrogen as SN 1993J.

While there is a small possibility that some other line is blended with $\mathrm{H} \alpha$ and comes to dominate the absorption feature at late times, we believe the simplest explanation is that it is due to $\mathrm{H} \alpha$ at all times. As an example, Deng et al. (2000) claimed that the increasing prominence of $\mathrm{C}_{\text {II }} \lambda 6580$ relative to $\mathrm{H} \alpha$ explained the time evolution of the $6300 \AA$ feature in the SN Ib 1999 dn, but Branch et al. (2002) presented arguments against the presence of strong $\mathrm{C}_{\mathrm{II}}$ in that particular object, including the weakness of any C II $\lambda 4745$ absorption. In addition, the lack of C I lines in the NIR spectra of SN 2008ax (see below) suggests that carbon likely does not make a strong contribution to the optical spectrum as well.

\subsubsection{Velocity Evolution}

Further evidence to support the $\mathrm{H} \alpha$ identification for the $6270 \AA$ feature in SN 2008ax can be seen from its velocity evolution, which we have listed in Table 3 and plotted in Figure 8 . Our velocity measurements for the absorption minima are derived by taking the wavelength of the minimum flux of a spline fit to the absorption profile and applying the relativistic Doppler formula for the velocity. Branch et al. (2002) used their SYNOW code to fit the spectra of a sample of $\mathrm{SNe}$ Ib and 
Table 3

Velocities of Absorption Minima

\begin{tabular}{|c|c|c|c|c|c|c|}
\hline $\begin{array}{l}\Delta t^{\mathrm{a}} \\
\text { (days) }\end{array}$ & $\begin{array}{l}t_{B_{\max }}{ }^{\mathrm{b}} \\
\text { (days) }\end{array}$ & $\begin{array}{c}\mathrm{H} \alpha \\
\left(\mathrm{km} \mathrm{s}^{-1}\right)\end{array}$ & $\begin{array}{c}\text { He I } \lambda 5876 \\
\left(\mathrm{~km} \mathrm{~s}^{-1}\right)\end{array}$ & $\begin{array}{c}\text { He I } \lambda 7065 \\
\left(\mathrm{~km} \mathrm{~s}^{-1}\right)\end{array}$ & $\begin{array}{l}\text { He I } 1.0830 \mu \mathrm{m} \\
\left(\mathrm{km} \mathrm{s}^{-1}\right)\end{array}$ & $\begin{array}{c}\text { He I } 2.0581 \mu \mathrm{m} \\
\left(\mathrm{km} \mathrm{s}^{-1}\right)\end{array}$ \\
\hline 2.34 & -16.5 & $-24,600$ & $-13,700$ & $\ldots$ & $\ldots$ & $\ldots$ \\
\hline 5.33 & -13.6 & $-19,400$ & $-11,800$ & $-11,100$ & $\ldots$ & $\ldots$ \\
\hline 6.12 & -12.8 & $-18,500$ & $-11,600$ & $-11,000$ & $\ldots$ & $\ldots$ \\
\hline 8.97 & -9.9 & $-15,500$ & $-10,500$ & -9100 & $\ldots$ & $\ldots$ \\
\hline 10.2 & -8.7 & $\ldots$ & $\ldots$ & $\ldots$ & $-11,700$ & $-10,000$ \\
\hline 11.2 & -7.7 & $\ldots$ & $\ldots$ & $\ldots$ & $-11,300$ & -9800 \\
\hline 14.1 & -4.8 & $-13,700$ & -9400 & -8300 & $\ldots$ & $\ldots$ \\
\hline 22.0 & 3.1 & $-13,600$ & -8700 & -7900 & $\ldots$ & $\ldots$ \\
\hline 40.2 & 21.3 & $\ldots$ & $\ldots$ & $\ldots$ & $-11,900$ & -9300 \\
\hline 41.0 & 22.1 & $-13,500$ & -8400 & -7700 & $\ldots$ & $\ldots$ \\
\hline 53.0 & 34.1 & $-13,500$ & -7600 & -7200 & $\ldots$ & $\ldots$ \\
\hline 56.2 & 37.3 & $\ldots$ & -7400 & -7200 & $\ldots$ & $\ldots$ \\
\hline 63.0 & 44.1 & $\ldots$ & -7200 & -7000 & $\ldots$ & $\ldots$ \\
\hline 66.0 & 47.1 & $\ldots$ & $\ldots$ & $\ldots$ & $-11,100$ & -9300 \\
\hline
\end{tabular}

Notes.

a Time after shock breakout.

b Time after maximum light in the $B$ band.

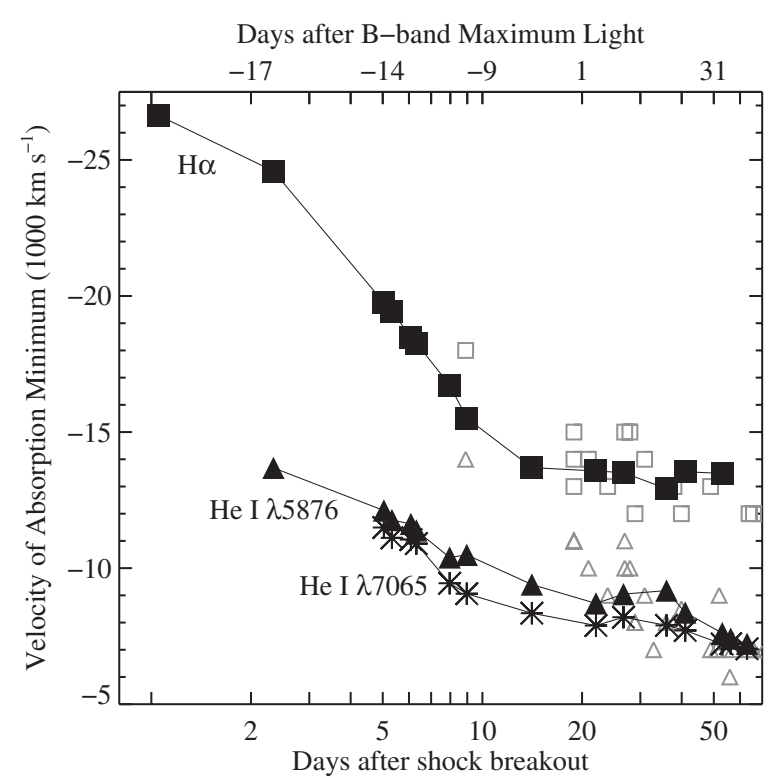

Figure 8. Velocities of the absorption minima of $\mathrm{H} \alpha$ (filled squares), $\mathrm{He}$ I $\lambda 5876$ (filled triangles), and He I $\lambda 7065$ (black stars) for SN 2008ax. The first $\mathrm{H} \alpha$ data point (at day 1.05) is from Blondin et al. (2008). Other data points in gray represent velocities for hydrogen (open squares) and $\mathrm{He}$ I (open triangles) for various SNe Ib taken from the SYNOW fits of Branch et al. (2002). The points from Branch et al. (2002) are listed relative to maximum light and have been shifted assuming the SNe all had the same rise time as SN 2008ax of 19 days. The velocities for SN 2008ax are consistent with those of the other objects in the literature.

derived minimum velocities for $\mathrm{H} \alpha$ and $\mathrm{He} \mathrm{I}$, which are plotted in Figure 8 as open gray boxes and triangles, respectively, assuming that the $6270 \AA$ feature is always due to $\mathrm{H} \alpha$. In order to plot the literature points on the same graph as SN 2008ax when we do not know the dates of explosion of the other objects, we have assumed a common rise time to maximum light, which is likely incorrect at the level of a few days offset per object due to the dispersion in $\mathrm{SN}$ Ib rise times. The post-maximum-light $\mathrm{H} \alpha$ and helium velocities in SN 2008ax are consistent with the normal SNe Ib.

The very high early-time velocity of the $\mathrm{H} \alpha$ absorption minimum in SN 2008ax rapidly declined until day 14, when it started to asymptotically approach a value of $-13,500 \mathrm{~km} \mathrm{~s}^{-1}$, which is well above the photospheric and helium velocities, but within the range of variation shown by the other objects. An identification of the $6270 \AA$ feature with either C II $\lambda 6580$ (e.g., Deng et al. 2000) or Si II $\lambda 6355$ (e.g., Hamuy et al. 2002) would have to explain the unusual asymptotic velocities $(-14,300$ and $-3900 \mathrm{~km} \mathrm{~s}^{-1}$, respectively) of those elements. Even if $\mathrm{H} \alpha$ were blended with absorptions from those ions, the late-time velocities are a strong argument that $\mathrm{H} \alpha$ must be dominant.

We have not plotted the $\mathrm{H} \beta$ velocities in Figure 8 because $\mathrm{H} \beta$ was completely dominated by Fe II lines after about day 9, but our last solid detection on day 9 also had an absorption minimum at about $-13,000 \mathrm{~km} \mathrm{~s}^{-1}$. The most natural explanation is that the $6270 \AA$ feature is indeed due to $\mathrm{H} \alpha$ and that it formed in a low-mass hydrogen-rich layer in the outer ejecta. The hydrogen velocities never dropped below $-13,000 \mathrm{~km} \mathrm{~s}^{-1}$ because there was no hydrogen interior to that in the ejecta.

Branch et al. (2002) also point out that lines forming at significantly greater velocities than the continuum photosphere (a geometry they term "detached" line formation) will have a characteristic profile with deep P-Cygni absorption and a weak emission component. This seems consistent with the unusually deep absorption components of the Balmer lines in SN 2008ax at early times (Figure 4) and the lack of an emission component to $\mathrm{H} \alpha$ at later times.

\subsubsection{NIR Spectra}

Next we consider the post-maximum-light evolution of our NIR spectra. The early-time spectra were relatively smooth with most features attributable to He I lines, but after maximum light 
many more features appeared, most of which are rather subtle blends of emission from neutral or singly ionized intermediatemass elements such as sodium, silicon, magnesium, and calcium (e.g., Meikle et al. 1989; Gerardy et al. 2004). The most prominent of these is an emission feature from $\mathrm{Mg}$ I at $1.503 \mu \mathrm{m}$. The strongest expected NIR lines from iron-peak elements are [Fe II] 1.257 and $1.644 \mu \mathrm{m}$ (Meikle et al. 1989). Our spectra do not show those features, but this may not be significant because our last epoch of observation (day 66) is before the full transition to the nebular phase and subsequent emergence of the forbidden lines. We defer detailed analysis of the relative contributions of each element to the blends to future work on the analysis of the NIR spectra of SNe Ib/c (G. H. Marion et al. 2011, in preparation).

We do wish to examine one issue, however, which is the relative strength of carbon and oxygen lines. OI lines, in particular $\lambda 7774, \lambda 9266$, and $1.129 \mu \mathrm{m}$, are present at late times in SN 2008ax (Figure 3). The strong Ca II NIR triplet absorption prevents us from determining the presence of any contribution from O I $\lambda 8446$. C I lines have been strongly detected in some SNe Ic, such as SN 1994I (Sauer et al. 2006) and SN 2007gr (Valenti et al. 2008; Hunter et al. 2009). However, most strippedenvelope SNe do not show such lines, including other SNe Ic such as SN 2002ap (Gerardy et al. 2004). The strong $C_{\text {I }}$ lines near $\lambda 9094, \lambda 9406, \lambda 9658$, and $1.069 \mu \mathrm{m}$ fall in the overlap region between our optical and NIR spectra of SN 2008ax and clearly are not present in either. Gerardy et al. (2004) identified an emission feature from $\mathrm{C}_{\mathrm{I}}$ at $1.454 \mu \mathrm{m}$ at 30 days after maximum light in the SN Ic 2000ew that was almost as strong as the neighboring $\mathrm{Mg}$ I $1.503 \mu \mathrm{m}$ emission line, but no such feature is visible in our SN 2008ax NIR spectra at comparable epochs (Figure 3). The reason why some SNe Ic have significantly stronger carbon lines than other strippedenvelope SNe remains unclear (Valenti et al. 2008; Hunter et al. 2009).

\subsection{Late-time Spectra}

After maximum light, the rapid spectral evolution seen at earlier times slowed down. P-Cygni features from $\mathrm{He}$ I lines $(4471,5015,5876,6678,7065$, and $7281 \AA$ ) all became prominent in the flux spectrum (Figure 1), and the emission component of the Ca II NIR triplet began to dominate the line profile. The absorption component of the NIR triplet largely disappeared between days 41 and 53, while the $\mathrm{H} \alpha$ absorption near $6270 \AA$ became hard to discern at the same time. These spectral changes coincide with the light curve of SN 2008ax transitioning from the main peak to the late-time radioactivedecay tail (P08; Roming et al. 2009). The late-time spectra of SN 2008ax, starting 85 days after explosion, are plotted in Figure 9. Over the next 70 days, the spectra make the transition from the photospheric to the nebular phase. The PCygni absorption features weaken and strong emission lines from oxygen and calcium start to dominate the spectrum.

For comparison, a spectrum of SN 1993J at similar epochs is also plotted. Balmer lines are still present in the SN 1993J spectrum. H $\alpha$ never disappeared from the SN 1993J spectra, even as $\mathrm{H} \alpha$ faded in the first several months after maximum light (Filippenko et al. 1994). H $\alpha$ subsequently rose in strength in SN 1993J as the interaction with hydrogen-rich CSM began, but our SN 2008ax spectra do not extend to such late times.

Any $\mathrm{H} \alpha$ emission at late times must be hidden in the far red wing of the $\left[\mathrm{O}_{\mathrm{I}}\right] \lambda \lambda 6300,6364$ line profile. Milisavljevic et al. (2010) do identify $\mathrm{H} \alpha$ in that red wing and there does appear

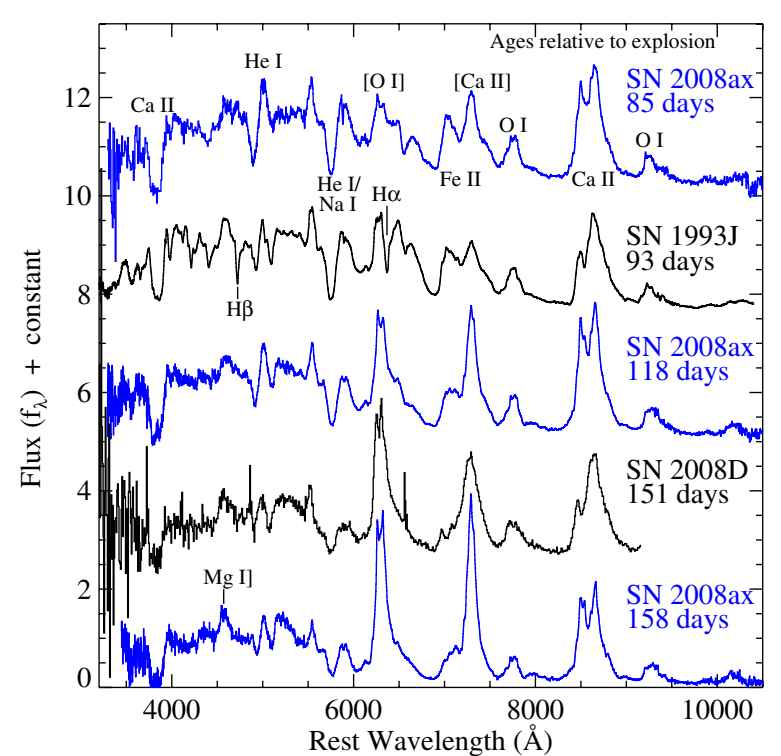

Figure 9. Late-time spectra of SN 2008ax during the transition to the nebular phase. Prominent spectral features are labeled. The spectrum of SN 1993J is from 1993 June 28 (Filippenko et al. 1994) and still shows evidence of hydrogen, with the Balmer absorption lines labeled. No strong hydrogen lines are evident in the SN 2008ax spectra or in the SN 2008D spectrum from 2008 June 7 (Modjaz et al. 2009). An additional total-flux spectrum of SN 2008ax was obtained on day 99 in spectropolarimetry mode and is discussed in Section 4.

(A color version of this figure is available in the online journal.)

to be emission with a box-like profile present in their day 307 spectrum, similar to SN 1993J at late times, albeit at a greatly reduced level. However, any $\mathrm{H} \alpha$ present at the epoch of our data must be blended with other lines, as there is little difference in the red wing of the [O I] line profile between SN 2008ax and the SN 2008D spectrum from a similar epoch (Modjaz et al. 2009). The biggest difference between those two objects at these late times is that SN 2008D shows broader emission lines. Electron scattering can also naturally explain red wings of the nebular line profiles (Fransson \& Chevalier 1989). In short, we do not find any compelling evidence for $\mathrm{H} \alpha$ emission in our spectra of SN 2008ax at late times. However, spectra taken at even later times do appear to show the emergence of box-like $\mathrm{H} \alpha$ emission indicative of CSM interaction (Milisavljevic et al. 2010; Maurer et al. 2010a; Taubenberger et al. 2011).

We have plotted the profiles of strong emission lines in our latest SN 2008ax spectrum, taken on day 158, in Figure 10. The oxygen lines collectively have rather different line profiles from each other and from the [Ca II] $\lambda \lambda 7291,7324$ emission. Interest in the late-time line profiles of stripped-envelope $\mathrm{SNe}$ has risen in recent years after Mazzali et al. (2005) identified double-peaked oxygen and magnesium lines in SN 2003jd and interpreted them as being due to an off-axis jet-like explosion. Subsequent work (Maeda et al. 2008; Modjaz et al. 2008; Taubenberger et al. 2009; Milisavljevic et al. 2010; Maurer et al. 2010 b) has shown that such profiles are ubiquitous, although the interpretation is uncertain. In particular, Milisavljevic et al. (2010) have presented an extensive study of the late-time emission-line profiles of SN 2008ax along with comparisons to several other stripped-envelope $\mathrm{SNe}$.

In the case of SN 2008ax, the [OI] $\lambda \lambda 6300,6364$ line profile is clearly double peaked, with the peaks separated by $\sim 3000 \mathrm{~km} \mathrm{~s}^{-1}$. The O I $\lambda 7774$ emission exhibits a flatter top, but with a definite emission peak under the bluer of the two peaks seen in $\left[\mathrm{O}_{\mathrm{I}}\right] \lambda \lambda 6300,6364$ (some of the weaker wiggles 


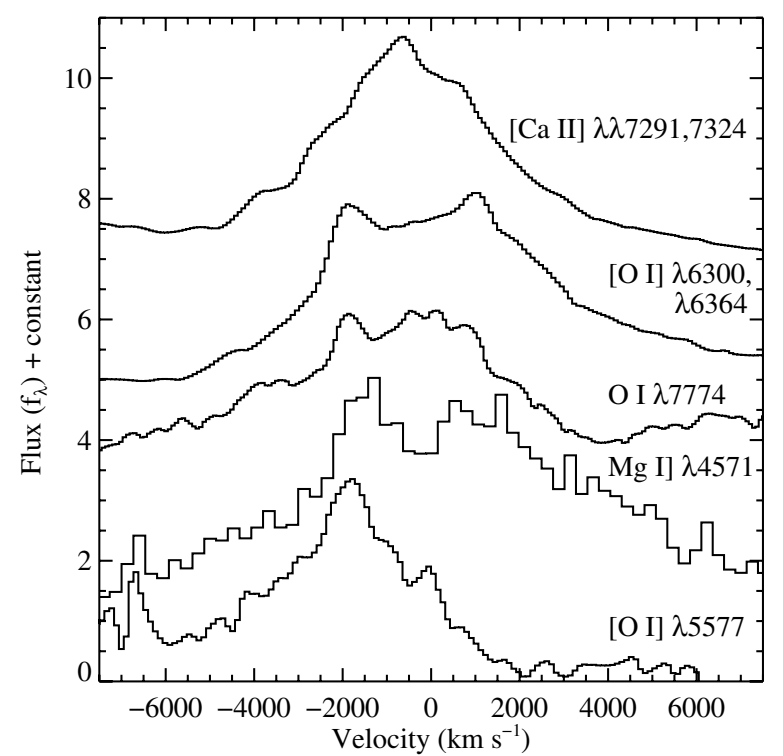

Figure 10. Late-time emission-line profiles of SN 2008ax on day 158, our latest epoch of observations. The [Ca II] line profile (presented relative to the average wavelength of the doublet of $7308 \AA$ ) is largely symmetrical. The [O I] $\lambda \lambda 6300$, 6364 emission (presented relative to $\lambda 6300$ ) is double peaked, but other oxygen lines do not share the same line profile.

seen in the O I $\lambda 7774$ profile are likely due to poor flat-fielding). The [O I] $\lambda 5577$ emission line has a single blueshifted peak, again at a radial velocity of $-2000 \mathrm{~km} \mathrm{~s}^{-1}$. Although our data at bluer wavelengths are very noisy, the $\mathrm{Mg}$ I] $\lambda 4571$ emission feature does appear to be double peaked as well. This represents a difference with the spectra presented by Milisavljevic et al. (2010). Their spectra showed a single blueshifted peak for the $\left.\mathrm{Mg}_{\mathrm{I}}\right] \lambda 4571$ line, similar to the [O I] $\lambda 5577$ emission at early times. However, their earliest spectrum including $\lambda 4571$ was taken on day 307 , potentially indicating that the line profile underwent significant evolution.

Milisavljevic et al. (2010) found that toroidal models for the oxygen-emitting zone had problems explaining the line profiles of most stripped-envelope SNe. They explored two alternatives in the case of SN 2008ax. The first invoked a blueshifted oxygen distribution that was optically thick so that $\lambda 6300$ and $\lambda 6364$ had a 1:1 ratio. The second had a largely symmetric broad oxygen distribution with an additional blueshifted clump producing the peaks in the profile. Both models still have some drawbacks, leaving the geometry highly uncertain. Our Mg I] $\lambda 4571$ line profile at the early nebular phase indicates that the magnesium distribution (which is thought to trace the oxygen distribution as well; Taubenberger et al. 2009) is not highly blueshifted, disfavoring the first model of Milisavljevic et al. (2010). Regardless of the actual geometry, these asymmetric and double-peaked oxygen line profiles are a sign of strong asphericities in the core of SN 2008ax.

\section{SPECTROPOLARIMETRY}

Thomson scattering in hot SN atmospheres polarizes outgoing radiation. Spatially unresolved SNe will show no net polarization if they are circularly symmetric when projected on the plane of the sky. However, if they are aspherical the net continuum polarization will be nonzero (Shapiro \& Sutherland 1982). Line opacity is generally thought to be depolarizing, so polarization modulations across spectral features can trace the aspherical distribution or excitation of individual chemical elements within the ejecta. The expectation is that line scattering in an axisymmetric, but aspherical, electron-scattering SN atmosphere will produce "inverted" P-Cygni polarization modulations (e.g., Jeffery 1991a). Unpolarized line emission near zero velocity dilutes the continuum polarization down to almost zero while selective blocking of forward-scattered continuum light results in a polarization maximum associated with the flux minimum.

Detailed analysis of the Stokes parameters $q$ and $u$ can also test whether any asphericities present are axisymmetric. In axisymmetry, data points representing bins in wavelength will lie along a line in the $q-u$ plane, as they represent greater or lesser amounts of polarization with a consistent orientation. A more complex distribution of wavelength bins in the $q-u$ plane is indicative of non-axisymmetric asphericities. In particular, if the wavelength bins associated with spectral lines from different ions have distinct behaviors, then the two ionic species likely have different spatial distributions within the ejecta. Thus, we will be able to compare the structure of the ejecta of SN 2008ax with those of previously studied stripped-envelope SNe (Wang \& Wheeler 2008). While we can examine the relative distributions of different chemical elements and make comparisons to other objects, spectropolarimetric data cannot be inverted to directly determine the actual geometry.

Core-collapse SNe occurring in progenitor stars that have retained most of their massive hydrogen envelopes explode as normal SNe II. Well-studied examples, such as the SN IIP 1999em (Leonard et al. 2001), show only small values of the polarization at early times while the photosphere recedes through the massive and apparently almost spherical hydrogen envelope. However, evidence is mounting that even those corecollapse explosions have highly aspherical cores (Leonard et al. 2006; Chornock et al. 2010). Wang et al. (2001) identified a trend where the observed amount of polarization in core-collapse $\mathrm{SNe}$ increases both with time, as the centers of the explosions become visible, and with increasing amounts of stripping of the progenitor stars. Therefore, it should not be a surprise that a SN whose progenitor was as highly stripped as that of SN 2008ax should show a strong polarization signal even at early times.

We have plotted $q$ and $u$ from our two epochs of earlytime (days 6 and 9) spectropolarimetry of SN 2008ax in Figure 11. High levels of polarization are present, with observed polarizations in the rest-frame $V$ band of $1.37 \pm 0.04 \%$ and $1.61 \pm 0.04 \%$ on days 6 and 9 , respectively. This change in polarization in only three days, along with the very large polarization modulations across spectral features such as $\mathrm{H} \alpha$, is evidence of substantial intrinsic polarization in SN 2008ax.

\subsection{Interstellar Polarization Correction}

We should expect that interstellar polarization (ISP) from dust in the host galaxy of SN 2008ax makes a significant contribution to the observed polarization, so we wish to remove this effect before studying its intrinsic polarization properties. Typical values for the polarization efficiency of Galactic dust (Serkowski et al. 1975) imply a potential host ISP of $1.5 \%$ for our adopted reddening. The Galactic component of the reddening should contribute negligibly to the ISP. In order to separate intrinsic SN polarization from ISP, we must make some assumptions. Early work on the SN IIb 1993J assumed that the intrinsic polarization in the emission component of $\mathrm{H} \alpha$ should be zero (Trammell et al. 1993b; Höflich et al. 1996; Tran et al. 1997). 

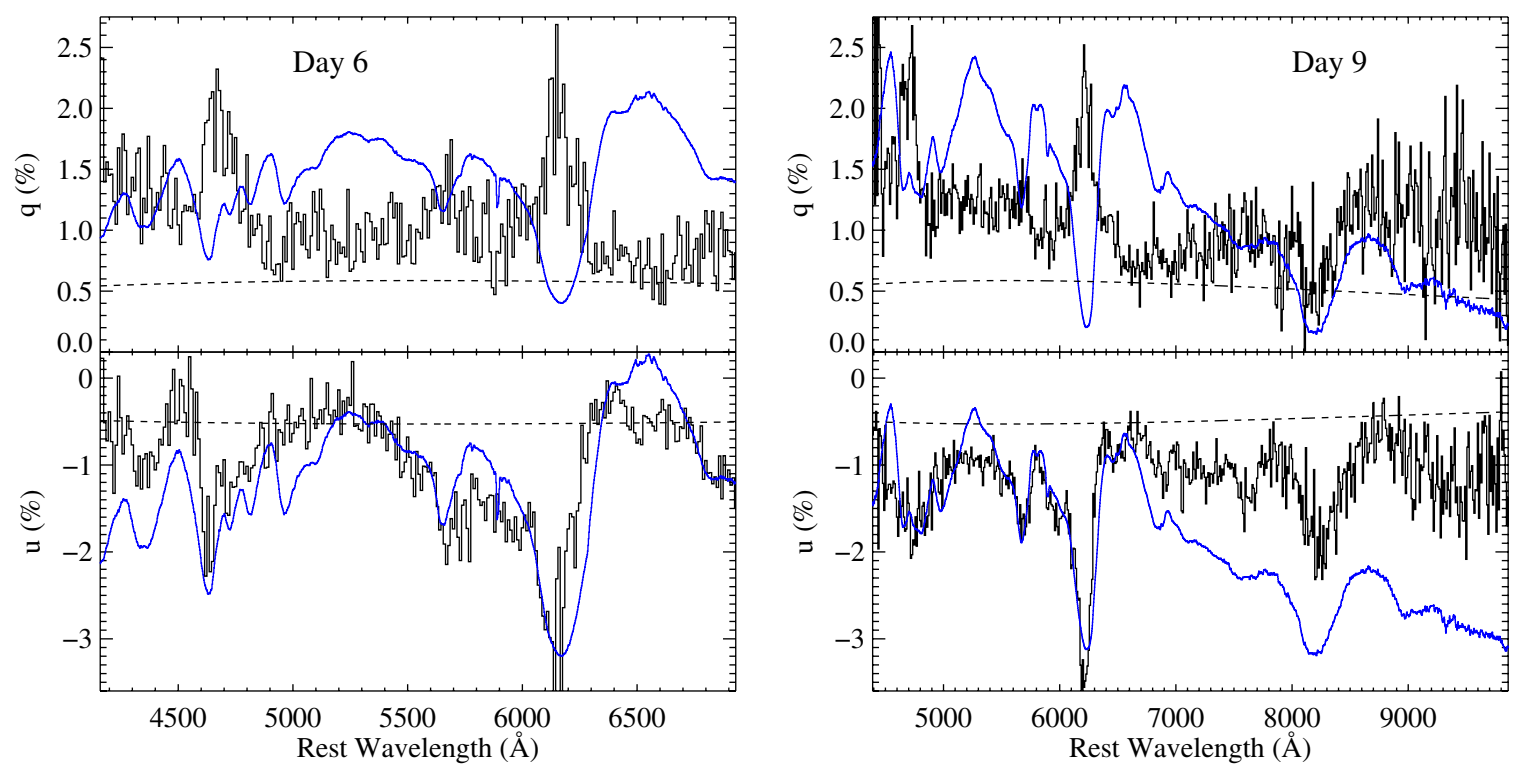

Figure 11. Early-time spectropolarimetry of SN 2008ax, uncorrected for ISP and plotted in $10 \AA$ bins. Day 6 is on the left and day 9 is on the right. The gray line in each panel (blue in the online edition) is the total-flux spectrum (in $f_{\lambda}$, with arbitrary multiplicative and additive offsets applied) from each night to guide the eye. The dashed lines show our preferred ISP as a reference. Strong polarization modulations are associated with the line features in the total-flux spectrum.

(A color version of this figure is available in the online journal.)

Maund et al. (2007b) showed that this assumption was false, at least in the case of the peculiar SN IIb 2001ig, in part due to blending of He I $\lambda 6678$ with the peak of $\mathrm{H} \alpha$. Our spectra of SN 2008ax at these early times (gray lines in Figure 11; blue in the online version) do show developing He I lines, so we will heed the cautionary example of SN 2001ig and use another method to estimate the ISP.

Maund et al. (2007b) were able to robustly measure the ISP to SN 2001ig from spectropolarimetry obtained on day 256. At that late epoch, the optical depth to electron scattering in the SN ejecta should be small as the expansion of the $\mathrm{SN}$ and consequent decrease in electron density results in geometric dilution of the scattering probability. As a consistency check, no polarization modulations were seen across SN spectral features, indicating that the measured polarization was entirely due to ISP. A similar method was successfully used by Jeffery (1991b) to correct the collected SN 1987A polarization data set for ISP.

Our final epoch of spectropolarimetry of SN 2008ax was taken on day 99 and is plotted in Figure 12. Strong polarization modulations are still seen at this late time, particularly across the Ca II NIR triplet emission feature near $8600 \AA$, indicating that there are still both substantial asphericities present in the ejecta and a sufficient optical depth to electron scattering to add a component of intrinsic SN polarization to the ISP to form the polarization signal we measure. The pattern of the polarization modulations, with decreases in polarization present at the strong emission lines (particularly in $q$ ), suggests a method to decompose the polarization.

We expect that the continuum polarization we see at this late time is due to photospheric continuum light scattering off electrons relatively deep in the ejecta. The strong emission lines which become more prominent as the SN transitions to the nebular phase are mostly due to material located exterior to the region of highest optical depth to electron scattering. The intrinsically unpolarized photons in the lines will travel unimpeded to the outside observer, acquiring a polarization only from the ISP along the line of sight. A similar dilution effect

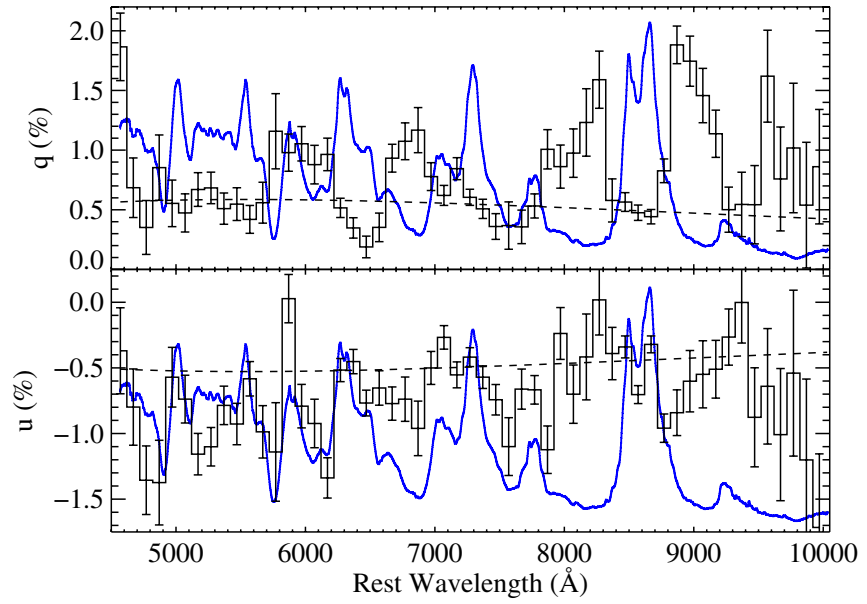

Figure 12. Polarization of SN 2008ax on day 99 after explosion, uncorrected for ISP. The two panels show the observed Stokes parameters $q$ (top) and $u$ (bottom) plotted versus wavelength compared to the total-flux spectrum of SN 2008ax (gray lines; blue in the online version). Depressions in polarization are visible at the wavelengths of the strong nebular emission lines, such as the Ca II NIR triplet near $8600 \AA$. The dashed lines are our preferred ISP, chosen to make the intrinsic polarization nearly zero at the peaks of the nebular emission lines. These variations in polarization across spectral features mean that SN 2008ax must have intrinsic continuum polarization in excess of $1 \%$ even at this late date, and hence large central asphericities.

(A color version of this figure is available in the online journal.)

was seen by Chornock et al. (2010) in the emission components of nebular lines such as [Ca II] in SNe IIP at late times.

With that in mind, we selected three wavelength regions of 6230-6350, 7250-7370, and 8470-8730 $\AA$ to represent the peaks of strong emission features from (respectively) [O I] $\lambda 6300,[\mathrm{Ca}$ II $] \lambda 7300$, and the Ca II NIR triplet. Integrating the observed polarization over those three windows gave consistent values for the Stokes parameters, with a spread of $\sim 0.1 \%$ in each, indicating that the peaks of the three lines do have similar polarizations of about $0.7 \%$. We fit an ISP curve to $20 \AA$ bins of 


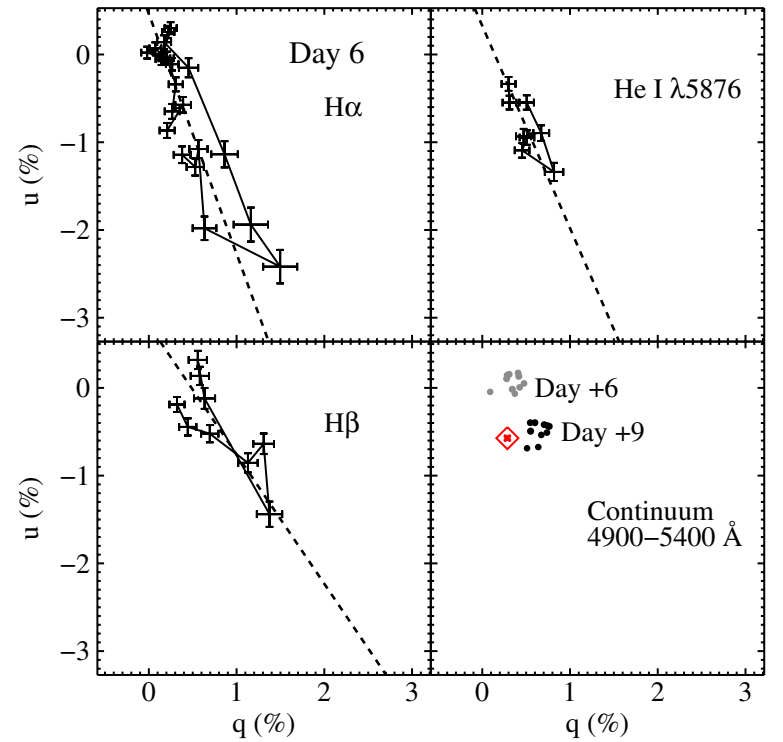

Figure 13. Line-polarization data from day 6 plotted in the $q-u$ plane, after subtraction of ISP. The data points with error bars in black in each panel represent $50 \AA$ bins in wavelength ranges corresponding to the indicated spectral features (H $\alpha: 5900-6950 \AA ; \mathrm{H} \beta: 4450-4900 \AA$; He I: 5450-5850 $\mathrm{A}$ ). The solid lines connect the points in order of wavelength to better show the loop structure. The overplotted dashed lines represent linear fits to the line-polarization data to guide the eye. The lower right panel shows a comparison of data points taken from the $4900-5400 \AA$ region on both nights, exhibiting a net shift of $0.64 \%$. Also plotted in that panel is the 7000-7450 $\AA$ continuum polarization from day 9 (diamond; red in the online version) for comparison with Figure 14.

(A color version of this figure is available in the online journal.)

the Stokes parameters from those three regions, following the functional form of Serkowski et al. (1975) with the modifications by Whittet et al. (1992). We fixed the peak wavelength of the ISP curve to $5550 \AA$ (or, equivalently, we set $R_{V}=3.1$ ) because the limited number of data points being fit were all at longer wavelengths than the expected peak, so the peak wavelength was not well constrained. Our measured values for $\left(q_{\mathrm{ISP}}, u_{\mathrm{ISP}}\right)$ are $(0.59 \%,-0.52 \%)$, which we adopt whenever we correct our data for ISP below, along with the functional form for ISP of Whittet et al. (1992). This ISP value has an uncertainty of $0.1 \%$ in each Stokes parameter based on the spread in the polarizations at the three line peaks, which is probably more realistic than the small formal errors from the fit (Maund et al. 2007b).

Figures 13 and 14 show wavelength intervals around important lines in the ISP-subtracted day 6 and 9 data in the $q-u$ plane. In addition to presenting the data in the $q-u$ plane, it is helpful to plot the ISP-subtracted spectropolarimetry versus wavelength. To do that, we first want to choose more optimal coordinates than Stokes $q$ and $u$, which are defined in reference to the position angle (P.A.) of north on the sky, which is unrelated to any property of the SN. The strong line-polarization modulations seen in Figure 11 appear as strong, almost linear features in the $q-u$ plane in Figures 13 and 14. Despite individually being almost linear in the $q-u$ plane, the line polarization features collectively are not aligned and prefer different polarization angles. This is a signature of strong deviations from axisymmetry and that the data are not well described by a single P.A., so there is no unambiguous choice of a preferred axis to use when rotating our coordinate system.

Inspection of Figure 11 reveals that the wavelength interval of $7000-7450 \AA$ on day 9 is devoid of strong line features in either the flux spectrum or the polarization. This region

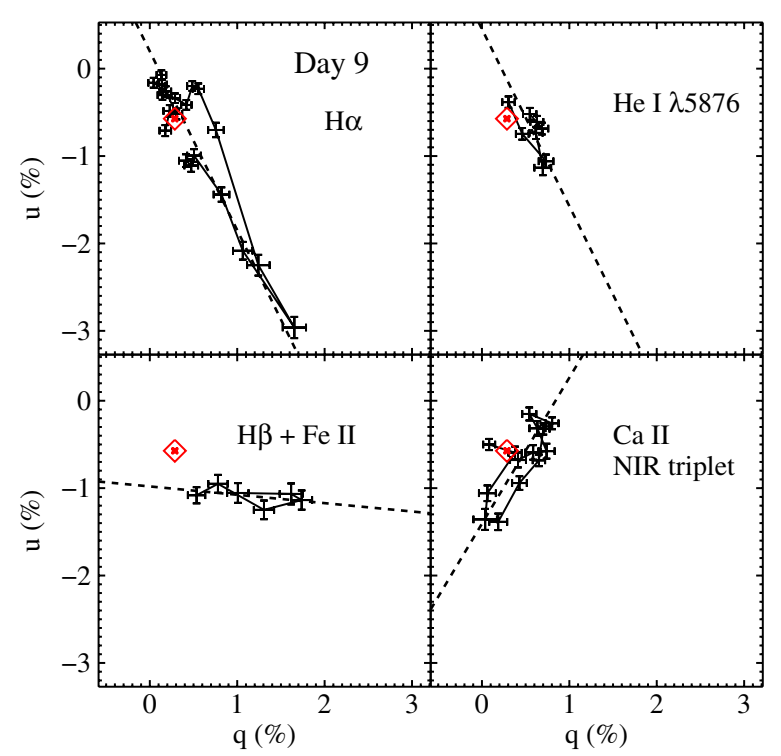

Figure 14. Line-polarization data from day 9 plotted in the $q-u$ plane, after subtraction of ISP. The diamond in each panel (red in the online version) represents the continuum polarization measured from the relatively line-free $7000-7450 \AA$ region (the error bar is smaller than the plot symbol). The data points with error bars in black in each panel represent wavelength bins corresponding to the indicated spectral features (H $\beta$ + Fe II: 4600-4900 $⿱$; He I: $5450-5850 \AA$; H $\alpha$ : 5950-6950 ̊; Ca II: 7900-9200 ̊̊). The solid lines connect the points in order of wavelength to better show the loop structure. The overplotted dashed lines represent linear fits to the line-polarization data to guide the eye. Between days 6 and 9, the $\mathrm{H} \alpha$ and $\mathrm{He}$ I $\lambda 5876$ polarization angles changed little. However, the polarization feature near $\mathrm{H} \beta$ is now dominated by blending with Fe II and has rotated to a different polarization angle reflecting the iron distribution. The Ca II data on day 9 show a polarization angle that is clearly different from that of the Balmer and He I lines.

(A color version of this figure is available in the online journal.)

falls between the P-Cygni absorption troughs from He I $\lambda 7065$ and $\mathrm{O}_{\mathrm{I}} \lambda 7774$, and we regard it as representative of the linefree continuum. The integrated ISP-subtracted polarization is $P=0.64 \pm 0.02 \%$ at a P.A. of $148.3 \pm 1.1$. This point is plotted as a diamond (red in the online version) in each panel of Figure 14. Interestingly, it falls near the dashed line showing a fit to the $\mathrm{H} \alpha$ data points. The ISP-subtracted polarization in the peak of the polarization feature associated with the $\mathrm{H} \alpha$ absorption trough on day $9(6190-6230 \AA)$ is $P=3.4 \pm 0.1 \%$ at an angle of $149.5 \pm 1.1$, consistent with the polarization angle of our adopted continuum region. The similar $\mathrm{H} \alpha$ polarization peak on day $6(6120-6180 \AA)$ has a polarization of $2.8 \pm 0.2 \%$ at an angle of $150.9 \pm 1.7$.

This excellent agreement between the continuum and $\mathrm{H} \alpha$ trough polarization angles (along with the polarizations of several other absorption lines; see below) gives us confidence that our value for the ISP was accurately determined and that the continuum P.A. (148.3) is physically significant in this object. We will choose a new coordinate system of rotated Stokes parameters (RSP; Trammell et al. 1993a; Tran 1995), which aligns $q_{\mathrm{RSP}}$ with this P.A. If the ejecta were axisymmetric, $q_{\mathrm{RSP}}$ would be a good estimator of the total polarization and $u_{\mathrm{RSP}}$ would be near zero. Deviations from zero in $u_{\mathrm{RSP}}$ are a signature of deviations from axisymmetry. An additional motivation for choosing RSP is to avoid the statistical bias of the formal polarization $\left(P=\left(q^{2}+u^{2}\right)^{\frac{1}{2}}\right)$ to positive values. The rotated spectropolarimetry is plotted versus wavelength in Figures 15 and 16. 


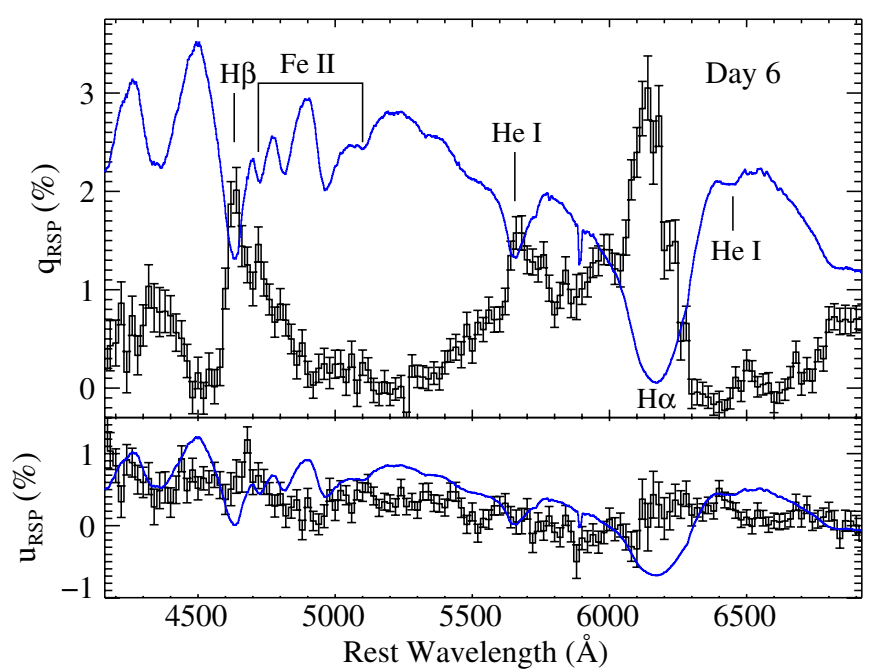

Figure 15. Day 6 spectropolarimetry corrected for ISP and rotated to align $q_{\text {RSP }}$ with the day 9 continuum polarization angle of $148^{\circ} .3$, presented in $20 \AA$ bins. Absorption minima of lines of polarimetric interest are labeled. If SN 2008ax were axisymmetric, $q_{\text {RSP }}$ would be an estimator of the total polarization, and $u_{\text {RSP }}$ would be centered on zero. The deviations of $u_{\text {RSP }}$ from zero are a sign of deviations from axisymmetry. Error bars on this and all subsequent plots are $1 \sigma$. (A color version of this figure is available in the online journal.)

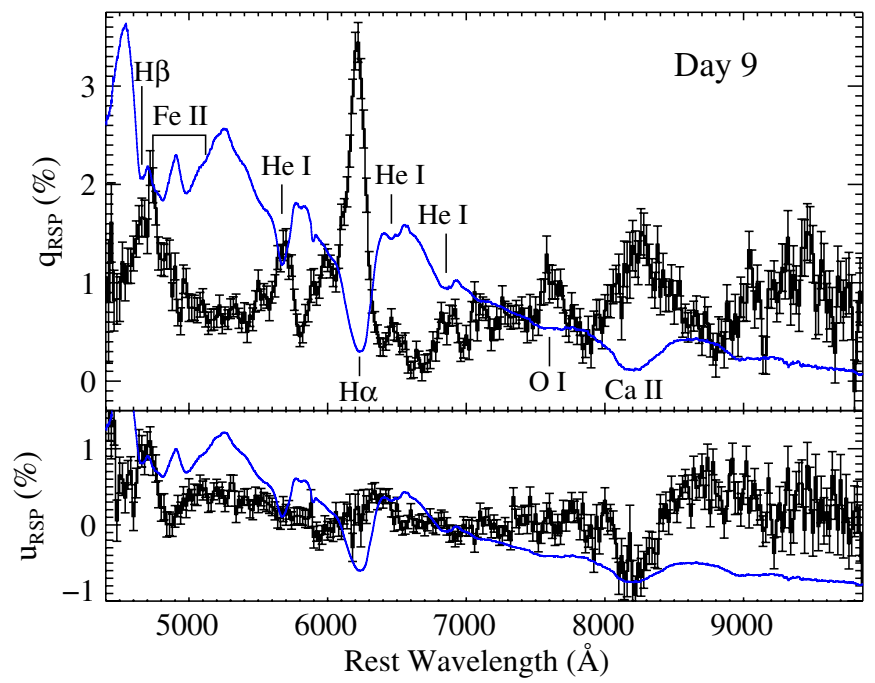

Figure 16. Day 9 spectropolarimetry corrected for ISP and rotated to align $q_{\mathrm{RSP}}$ with the 7000-7450 Å continuum polarization angle of 148.3 , presented in $20 \AA$ bins. Strong polarization modulations are seen in association with each P-Cygni feature. The absorption minima of lines discussed in the text are labeled. (A color version of this figure is available in the online journal.)

\subsection{Line Polarization}

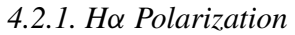

We will start our exploration of the line-polarization behavior of SN 2008ax with $\mathrm{H} \alpha$ because it is the strongest line in the spectrum and can be used as a reference. In addition to the full spectropolarimetry in Figures 15 and 16, we have plotted $q_{\mathrm{RSP}}$ and $u_{\mathrm{RSP}}$ versus velocity for $\mathrm{H} \alpha$ and $\mathrm{H} \beta$ in Figures 17 and 18.

Ignoring for the moment the modulations in $u_{\mathrm{RSP}}$, the basic shapes of the $\mathrm{H} \alpha$ polarization features on days 6 and 9 match the "inverted" P-Cygni polarization features discussed above. To test the expectation of low polarization near the line peak at zero velocity (or alternatively, our choice of ISP), we integrated the polarization over $6600-6700 \AA$ to estimate the $\mathrm{H} \alpha$ peak polarization, while avoiding most of the effects of the $\mathrm{He}$ I $\lambda 6678$

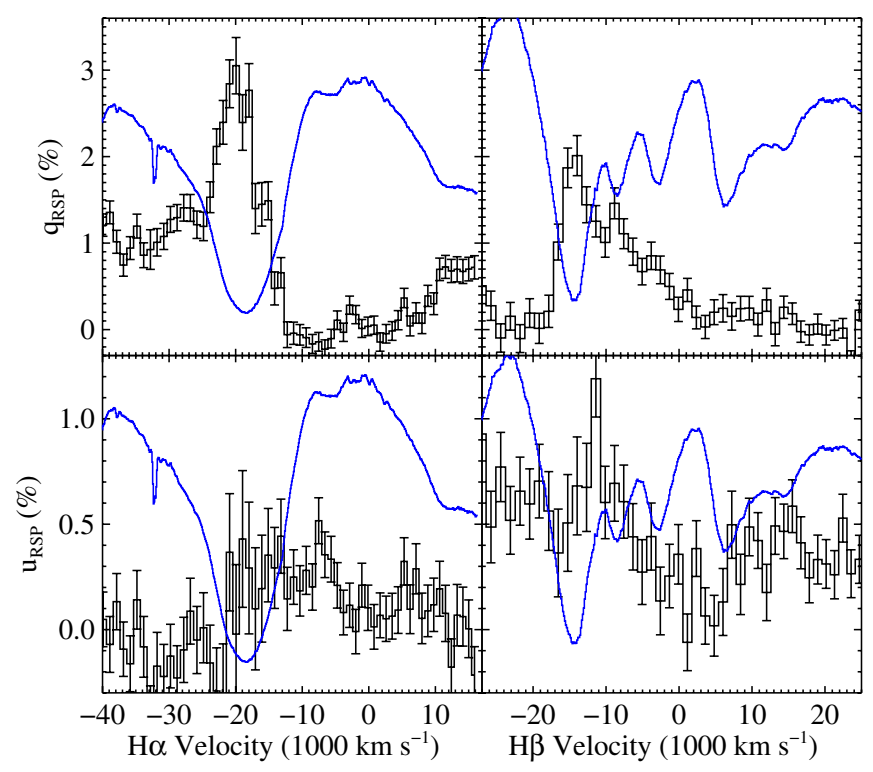

Figure 17. $\mathrm{H} \alpha$ and $\mathrm{H} \beta$ line polarization on day 6 versus velocity. The gray line in each panel (blue in the online version) is the total-flux spectrum plotted to guide the eye. The absorption near $-14,000 \mathrm{~km} \mathrm{~s}^{-1}$ in the panels on the right is due to $\mathrm{H} \beta$. The notches redward of that are due to Fe II multiplet 42. The polarization peaks in $q_{\mathrm{RSP}}$ are associated with the absorption minima of $\mathrm{H} \alpha$ and $\mathrm{H} \beta$.

(A color version of this figure is available in the online journal.)

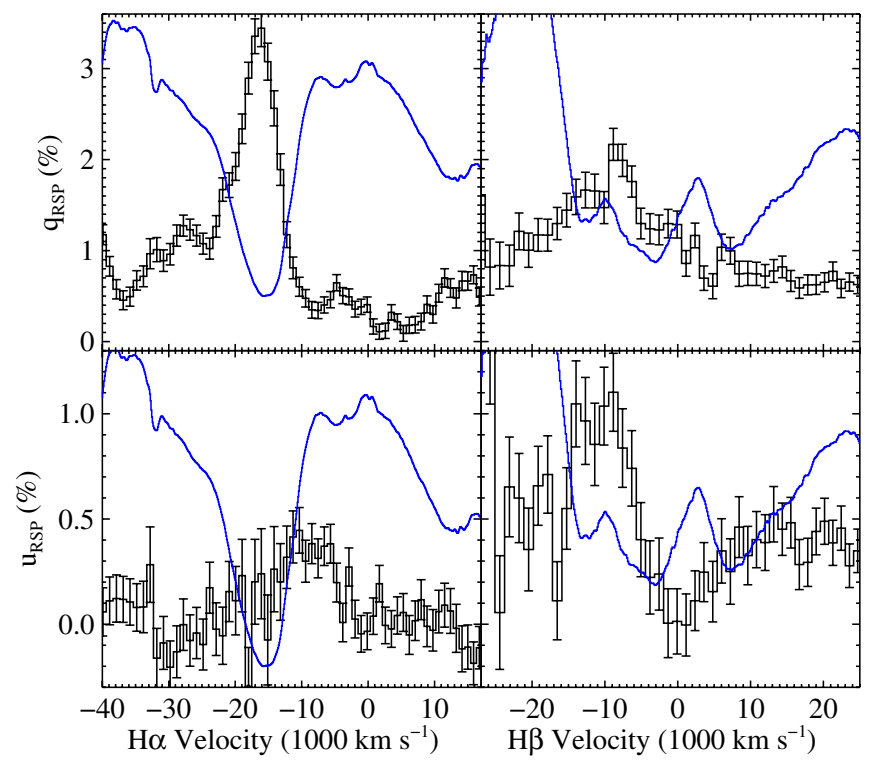

Figure 18. $\mathrm{H} \alpha$ and $\mathrm{H} \beta$ line polarization on day 9 versus velocity. The gray line in each panel (blue in the online version) is the total-flux spectrum plotted to guide the eye. $\mathrm{H} \beta$ is not prominent in the total-flux spectrum at this time (it is the absorption near $-13,000 \mathrm{~km} \mathrm{~s}^{-1}$ in the panels on the right) and the Fe II lines dominate the observed polarization signal.

(A color version of this figure is available in the online journal.)

absorption (Maund et al. 2007b). We obtain values for ( $q_{\mathrm{RSP}}$, $\left.u_{\mathrm{RSP}}\right)$ of $(0.06 \pm 0.05 \%, 0.12 \pm 0.05 \%)$ and $(0.17 \pm 0.04 \%$, $0.03 \pm 0.04 \%$ ) on days 6 and 9 , respectively. These low values for the $\mathrm{H} \alpha$ peak polarization despite some contamination from He $\mathrm{I} \lambda 6678$ give us additional confidence that our derived ISP is reasonable.

Similar peaks in polarization can be seen in $q_{\mathrm{RSP}}$ associated with most of the P-Cygni absorption features in Figures 15 and 16. In particular, there are clear features at $\mathrm{H} \beta$, the Fe II lines near $5000 \AA$, He I $\lambda 5876$, O I $\lambda 7774$, and the Ca II NIR 
triplet, as well as weaker peaks due to $\mathrm{He}$ I $\lambda 6678$ and $\lambda 7065$. In a purely axisymmetric system, all of the line features would show the inverted P-Cygni features in $q_{\mathrm{RSP}}$ but no modulation in $u_{\mathrm{RSP}}$, which is in clear conflict with the strong features seen in $u_{\mathrm{RSP}}$ for some lines in Figures 15 and 16. This is not an artifact of a poor ISP correction or coordinate rotation. The $q-u$ linepolarization plots in Figures 13 and 14 show that different lines have quite different polarization behaviors which are not aligned and indicate that the geometry of the ejecta of SN 2008ax was quite complex.

In addition to the evidence for deviations from axisymmetry seen from the ensemble of line features, individual lines show that the spatial distribution or excitation of individual chemical elements is complex. For example, the $\mathrm{H} \alpha$ data points in the upper left panel of Figure 13 are connected in order of wavelength by a line. If the $\mathrm{H} \alpha$ geometry were axisymmetric, the points would fall in a line. However, the modulation seen in $u_{\mathrm{RSP}}$ manifests itself in this plot as a two-dimensional spread of the $\mathrm{H} \alpha$ points. This spread is not random, but shows a systematic trend with wavelength in a manner consistent with the $q-u$ "loops" first identified by Cropper et al. (1988) in spectropolarimetry of SN 1987A. Loops are not confined to $\mathrm{H} \alpha$; all the lines plotted in Figures 13 and 14 show evidence for loop-like structure.

Chugai (1992) proposed that individual clumps of ${ }^{56} \mathrm{Ni}$ could result in $\mathrm{H} \alpha$ excitation asymmetries that produce wavelengthdependent polarization angle rotations across the line profile. While the loops in SN 1987A were seen in $\mathrm{H} \alpha$ and Ca II, subsequent work has found them to be ubiquitous in corecollapse SNe (Wang \& Wheeler 2008). The interpretation of loops and their implied velocity-dependent polarization angles has remained obscure. One possibility is that the clumpiness of the ejecta results in each isovelocity surface intersecting a somewhat different spatial distribution of each chemical element. However, if the clumps only existed on small scales, the polarization angles in different velocity bins would be essentially independent. The systematic rotation of the polarization angle with velocity implies that the relevant asphericities are coherent on larger scales.

Kasen et al. (2003) investigated the loop seen in the Ca II NIR triplet of the SN Ia 2001el and found that loops were generically produced by a line-scattering region with a misaligned axis of symmetry relative to the continuum and therefore that inversion of the observed Stokes parameters for a single object was highly underconstrained. Despite SN 2001el being an SN Ia, the models of Kasen et al. (2003) are surprisingly relevant to SN 2008ax, at least for $\mathrm{H} \alpha$. In SN 2001el, the geometry involved a highvelocity absorption from $\mathrm{Ca}$ II detached above the photosphere. As discussed above, the $\mathrm{H} \alpha$ line in SN 2008ax is also detached from the photosphere in a high-velocity shell. The misaligned aspherical shell models of Kasen et al. (2003) may therefore be quite relevant. With the long axis of the $\mathrm{H} \alpha$ loop being largely aligned with the continuum polarization, the $\mathrm{H} \alpha$ distribution likely has only a slight misalignment with the symmetry axis of the photosphere.

While the models of Kasen et al. (2003) may be able to explain loops seen in detached lines like $\mathrm{H} \alpha$, the relevance of these models to lines forming closer to the photosphere (such as $\mathrm{He}$ I) is less clear. An additional difficulty is with the amplitude of the polarization modulation. A polarization of $3.4 \%$ in the $\mathrm{H} \alpha$ line is very high, particularly for an $\mathrm{SN}$ being observed so soon after explosion. Similarly large polarizations $(P \lesssim 4 \%)$ were seen in the trough of the Ca II NIR triplet in SN 1987A (Cropper et al.
1988; Jeffery 1991a, 1991b), but only at late times (day 100). Jeffery (1991a) was unable to successfully model such a large line polarization. He invoked a special excitation asymmetry, especially to explain the differences between $\mathrm{H} \alpha$ and $\mathrm{Ca}$ II at that time.

Maund et al. (2007a) found similarly high polarization values in the $\mathrm{Ca}$ II lines in the peculiar SN Ib/c 2005 bf before maximum light and Maund et al. (2009) observed large $(P \approx 2 \%)$ calcium polarizations in SN 2008D. A common thread is that at the times of large line polarization, the lines in question formed at high velocities and were detached from the photosphere. Evidently, such a high-velocity, detached-shell geometry is quite favorable for the production of significant line polarization. That the lines showing this effect are frequently due to calcium may just be due to the relative strength of calcium lines in $\mathrm{SN}$ ejecta material at the low densities of most high-velocity material.

One last factor to mention in the context of very strong line-polarization values is deviations of the geometry of the high-velocity material from a spheroid. Partial photospheric obscuration at different velocity surfaces is responsible for the line polarization seen from a detached aspherical shell (Kasen et al. 2003), but the polarizing effectiveness is limited by the spheroidal geometry. If the absorbing material has a geometry far from that of a spheroid, such as a toroid or large clumps, then partial obscuration can very effectively generate large polarizations (Kasen et al. 2003). Such non-spheroidal geometries could also result in the appearance of the $\mathrm{H} \alpha$ line being quite different in strength or velocity along other lines of sight.

\subsubsection{Other Lines}

The polarization near $\mathrm{H} \beta$ is more complicated than at $\mathrm{H} \alpha$. On day 6 , the locus of points in the $q-u$ plane (Figure 13) is not linear or a simple loop. The long axis of the distribution is largely aligned with the $\mathrm{H} \alpha$ orientation, but not exactly. The peak polarization at the absorption minimum, integrated over $4610-4650 \AA$, is $P=1.99 \pm 0.14 \%$ at a P.A. of $157 \pm 2^{\circ}$, which is slightly different from that at $\mathrm{H} \alpha$. The right-hand panels of Figure 17 show that the polarization peak is centered on the absorption minimum. Unlike $\mathrm{H} \alpha, q_{\text {RSP }}$ does not go to zero near zero velocity. Instead, $q_{\mathrm{RSP}}$ remains high at velocities redward of the absorption minimum. Similarly, the polarization in $u_{\mathrm{RSP}}$ is elevated above zero. We interpret this behavior as being due to polarization induced by the superposed Fe II lines of multiplet 42 , which are visible as the three notches in the totalflux spectrum redward of $\mathrm{H} \beta$ in Figure 17.

The spectrum of SN 2008ax was undergoing rapid evolution at this time. By our next epoch of spectropolarimetry, day 9, both the spectrum and polarization near $\mathrm{H} \beta$ had changed significantly. The total-flux spectrum in the right-hand panels of Figure 18 shows that the absorption from $\mathrm{H} \beta$ had significantly weakened as the photosphere had receded through the hydrogen envelope. Meanwhile, the neighboring Fe II lines had strengthened. The polarization data points from this region are plotted in the lower left panel of Figure 14. The polarization feature clearly is no longer oriented similarly to $\mathrm{H} \alpha$ and prefers another direction which presumably reflects the iron distribution. The polarization in the core of the $\mathrm{H} \beta$ absorption (integrated over $4630-4680 \AA$ ) is $P=1.94 \pm 0.12 \%$ at a P.A. of $163 \pm 2^{\circ}$, but the polarization angle changes rapidly to the red as the effects of the Fe II lines manifest themselves.

The other lines shown in Figures 13 and 14 also exhibit loops. The long axis of the He $\mathrm{I} 25876$ loop is largely aligned with 
the continuum and $\mathrm{H} \alpha$ polarization axis, although the loop is responsible for some deviation from that axis. The amplitude of the modulation across $\lambda 5876$ is about $0.9 \%$ on day 9 . The polarization peak on day $6(5630-5700 \AA)$ has a polarization of $1.47 \pm 0.08 \%$ at a P.A. of $150.7 \pm 1.6$, evolving on day $9(5640-5740 \AA)$ to a polarization of $1.30 \pm 0.06 \%$ at a P.A. of $151.2 \pm 1.3$. The P.A. on both nights is consistent with the $\mathrm{H} \alpha$ and continuum P.A. The weaker modulations $(\Delta P \approx 0.3 \%)$ associated with He I $\lambda 6678$ and $\lambda 7065$ also appear to be mostly in the direction of $q_{\mathrm{RSP}}$, although the lower significance of those features prevents us from studying any substructure.

The loop formed by the Ca II NIR triplet feature on day 9 is clearly not aligned with any other ion in the spectrum or with the continuum. This result is independent of any ISP subtraction or rotation of coordinates. The relatively small wavelength dependence of ISP means that ISP subtraction approximates a shift of the origin of the $q-u$ plane. The differing behaviors of $\mathrm{H} \alpha$ and the NIR triplet can also be seen in the uncorrected $q$ and $u$ spectropolarimetry plotted in the right-hand panel of Figure 11. While the $\mathrm{H} \alpha$ trough polarization deviates from the continuum polarization in the positive $q$, negative $u$ direction, the NIR triplet absorption deviates in the negative $q$, negative $u$ direction. The net polarization modulation, or the length of the long axis of the loop, is about $1.3 \%$.

A small polarization increase is evident in the OI $\lambda 7774$ absorption trough in Figure 16, although the noise is too high relative to the amplitude of the feature to see a loop if one were present. The integrated polarization over the 7550-7700 interval is $\left(q_{\mathrm{RSP}}, u_{\mathrm{RSP}}\right)=(0.97 \%, 0.03 \%)$, with error bars of $0.06 \%$ on each Stokes parameter. This means that the polarization in the oxygen absorption is elevated by $0.33 \%$ above the continuum level, but has the same P.A.

In summary, the hydrogen, helium, and oxygen lines in SN 2008ax all have largely consistent orientations (ignoring the small modulations in $u_{\mathrm{RSP}}$ due to the loops), which they share with the continuum at red wavelengths. It is natural to identify this angle with the symmetry axis of the SN ejecta. Calcium and iron appear to have different orientations. SN 2008ax is not unique in this respect (Maund et al. 2007a, 2009), which we will discuss further below.

\subsection{Late-time Polarization}

We have also corrected our late-time spectropolarimetry for ISP, but we have chosen a new coordinate system to present the data. Regions of high continuum polarization are evident in Figure 12 between the nebular emission lines. We chose two such regions on either side of the Ca II NIR triplet, 7850-8250 and 8850-9150 $\AA$, and integrated the ISP-subtracted Stokes parameters. We obtained polarization angles of $174 \pm 5^{\circ}$ and $175 \pm 3^{\circ}$, respectively. We chose to rotate our new coordinate system $\left(q_{\mathrm{RSP} 2}, u_{\mathrm{RSP} 2}\right)$ to this preferred axis (weighted average of 174.3) and plotted the data in Figure 19. This method places most of the highest continuum polarization peaks into $q_{\mathrm{RSP} 2}$. If we had used the other rotated coordinate system, $u_{\mathrm{RSP}}$ would have shown large deviations from zero.

The basic pattern of high continuum polarization still holds in $q_{\mathrm{RSP} 2}$. The region of highest polarization is our continuum window of $8850-9150 \AA$, which has a net ISP-corrected polarization of $1.28 \pm 0.11 \%$, indicative of large asphericities in the inner core of the $\mathrm{SN}$ ejecta. The polarization dips down to nearly zero at the wavelengths of strong emission lines by construction due to our choice for the ISP.

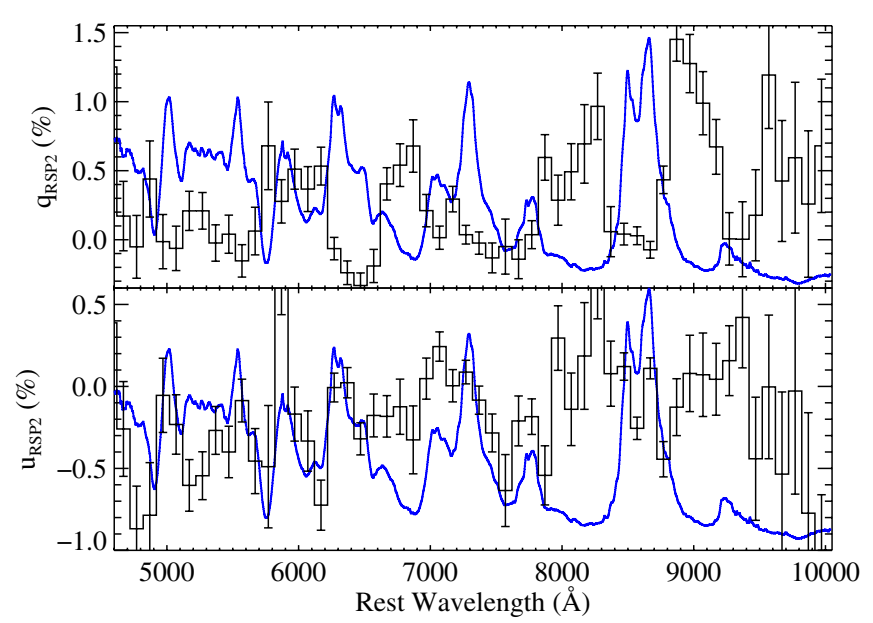

Figure 19. Day 99 spectropolarimetry after correction for ISP, and presented in $100 \AA$ bins. The data have been rotated to a new coordinate system ( $q_{\mathrm{RSP} 2}$, $u_{\mathrm{RSP} 2}$ ), chosen to align $q_{\mathrm{RSP} 2}$ with the strong continuum polarization signal near 8000 and $9000 \AA$

(A color version of this figure is available in the online journal.)

Curiously, the polarization at bluer wavelengths is nearly zero in $q_{\mathrm{RSP} 2}$, but is systematically negative in $u_{\mathrm{RSP} 2}$. The integrated polarization over 5050-5950 $\AA$ (approximating the $V$ band) is $P=0.26 \pm 0.06 \%$ at a P.A. (prior to rotation) of $143 \pm 5^{\circ}$. This P.A. is completely consistent with that of the continuum polarization angle measured on day 9 and used to rotate into our original $\left(q_{\mathrm{RSP}}, u_{\mathrm{RSP}}\right)$ coordinates, but is inconsistent with the P.A. in our redder continuum windows.

Usually the consistency of the continuum polarization angle from early to late times would be interpreted as resulting from a single axis of symmetry in the ejecta, but the rotation of the continuum polarization angle with wavelength at late times shows that the inner ejecta do not have a simple axisymmetric geometry. While such a rotation is potentially a symptom of poor ISP removal, the substantial scatter in the $q-u$ plane we observe shows that no single ISP could align the wavelengthdependent continuum angle. Perhaps the continuum polarization angle at late times is affected by individual clumps of ${ }^{56} \mathrm{Ni}$ producing regions of enhanced electron scattering (Chugai 1992). The $V$-band polarization angle could also be modified by the spatial distribution of the iron lines mostly responsible for the pseudocontinuum at those wavelengths.

Lastly, we note that one potential alternative method for determining the ISP would be to assume that the polarization at blue wavelengths is nearly zero at late times under the hypothesis that the large amount of Fe II opacity should result in complete depolarization of the continuum (e.g., Howell et al. 2001). The low $V$-band polarization we do measure after subtraction of our preferred ISP shows that this method would result in only a slightly different ISP subtraction.

If we had used the late-time $V$-band polarization to measure the ISP and then rotated our early-time data into a coordinate system aligned with the day 9 continuum polarization, there would be a few negative consequences. Specifically, the $\mathrm{H} \alpha$ line polarizations in our new $q_{\text {RSP }}$ vector would be somewhat offset such that the polarization peaks in the absorption troughs would still be at positive values of $q_{\mathrm{RSP}}$ but the polarization at the line peaks would be at negative values of $q_{\text {RSP }}$. Such a polarization profile is certainly possible (e.g., Jeffery 1989), but we prefer the result of our chosen ISP, making the polarization of the emission-line peaks at late times and in the $\mathrm{H} \alpha$ peaks at 
early times nearly zero. A small residual polarization in the $V$ band at late times may indicate that the iron responsible for the line opacity and depolarization of the continuum might not be physically located exterior to the continuum formation region and is instead cospatial with the scattering electrons such that some polarized flux can escape to the distant observer.

\section{DISCUSSION}

\subsection{Hydrogen Envelope}

The well-sampled spectroscopic sequences presented in Section 3 and by P08 and Taubenberger et al. (2011) demonstrate that SN 2008ax made a transition from the hydrogen-dominated spectra of an SN II to the helium-dominated spectra of an SN Ib, reflecting a progenitor that had lost most but not all of its hydrogen envelope (Crockett et al. 2008). A key question is how much hydrogen the progenitor retained at the time of explosion. The progenitor system of the prototypical SN IIb 1993J has been directly detected (Aldering et al. 1994; Maund et al. 2004; Maund \& Smartt 2009) and determined to have been a K-type supergiant interacting with a B-type supergiant companion. The direct observations of the progenitor of SN 2008ax are still ambiguous due to possible contamination from other stars and await final, late-time template images (Crockett et al. 2008). However, the progenitor of SN 2008ax was probably not similar to that of SN 1993J, and was either an even more highly stripped star in an interacting binary (superposed on a stellar cluster) or a W-R star with a small residual hydrogen envelope (a WNL/WNH star; Crockett et al. 2008), although Smith \& Conti (2008) argue that the WNH stars are still undergoing core $\mathrm{H}$ burning. The $\mathrm{W}-\mathrm{R}$ progenitor model may also have difficulty in explaining the shape of the bolometric light curve of SN 2008ax (Crockett et al. 2008). Chevalier \& Soderberg (2010) have recently proposed that SN 2008ax is one of a group of SNe IIb coming from compact W-R-like progenitors, while SN 1993J is the exemplar of a different class of $\mathrm{SNe}$ IIb that appear to come from more extended progenitors.

Ryder et al. (2004) observed modulations in the radio light curve of the SN IIb 2001ig, which they attributed to the interaction between the W-R wind of the progenitor and a hot companion, a conclusion reinforced when Ryder et al. (2006) found a supergiant star at the location of the SN long after explosion, which they identified as the companion to the progenitor. Soderberg et al. (2006) instead preferred to interpret the variations in the radio light curve as signs of a variable wind from a single massive $\mathrm{W}-\mathrm{R}$ star. These results are relevant to SN 2008ax because its radio light curve (Roming et al. 2009) shows similar modulations to those of SN 2001ig, although the pre-SN mass-loss rate of the SN 2008ax progenitor appears to have been lower than in other SNe IIb (Roming et al. 2009).

Spectroscopically, it seems clear that SN 2008ax had significantly less hydrogen in its envelope than SN 1993J. The Balmer lines in the optical spectra of SN 2008ax disappeared at a much earlier epoch than in SN 1993J (Section 3; P08), while the NIR spectra never showed much evidence for hydrogen. Typical models for the progenitor of SN 1993J (Shigeyama et al. 1994; Woosley et al. 1994) find a hydrogen-envelope mass of 0.2-0.9 $M_{\odot}$, with Houck \& Fransson (1996) measuring $\sim 0.3 M_{\odot}$ of hydrogen directly from the late-time spectra.

Elmhamdi et al. (2006) obtained spectroscopic hydrogenmass estimates for several stripped-envelope $\mathrm{SNe}$ using some simple assumptions. They derive $0.7 M_{\odot}$ of hydrogen for SN 1993J (on the high side of other estimates), but only $0.1 M_{\odot}$

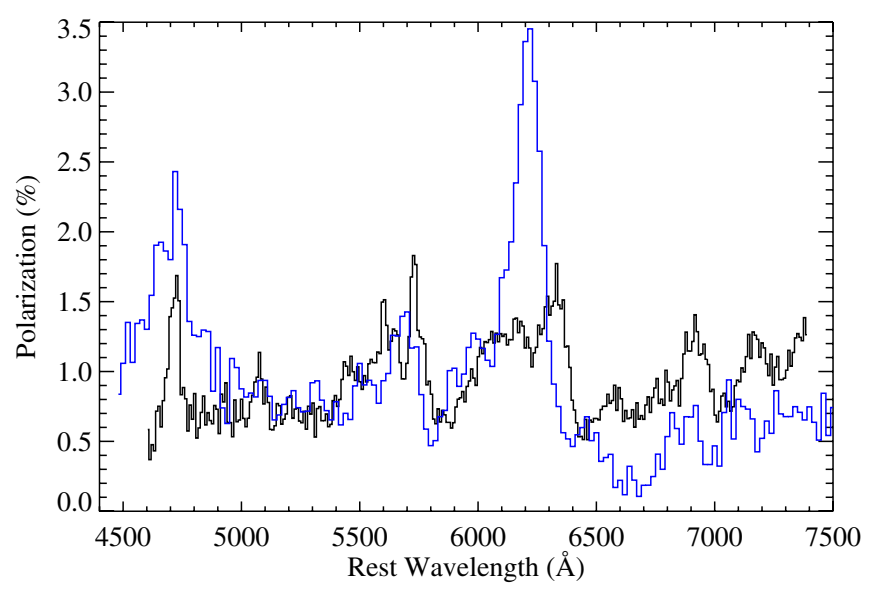

Figure 20. Day 9 polarization data for SN 2008ax (gray; blue in the online version) compared to the SN 1993J data (black) from 1993 April 30 after correction for ISP (Tran et al. 1997).

(A color version of this figure is available in the online journal.)

for the SN IIb (or hydrogen-rich SN Ib) $2000 \mathrm{H}$ (Branch et al. 2002), which had stronger absorption from $\mathrm{H} \alpha$ at late times than SN 2008ax (Figure 7). Branch et al. (2002) found that masses of order $10^{-2} M_{\odot}$ of hydrogen would be necessary in local thermodynamic equilibrium (LTE) to produce an optical depth in $\mathrm{H} \alpha$ of order unity near maximum light in SNe Ib. Tominaga et al. (2005) estimated that $\sim 0.02 M_{\odot}$ of hydrogen at high velocities would reproduce the observed $\mathrm{H} \alpha$ absorption in the $\mathrm{SN} \mathrm{Ib/c} \mathrm{2005bf,} \mathrm{which,} \mathrm{although} \mathrm{peculiar} \mathrm{and} \mathrm{differing}$ from SN 2008ax in other ways, was the closest spectroscopic match to SN 2008ax near maximum light. The broad-lined SN IIb 2003bg had stronger $\mathrm{H} \alpha$ absorption at late times than SN 2008ax (Hamuy et al. 2009), which Mazzali et al. (2009) were able to model using $\sim 0.05 M_{\odot}$ of hydrogen. Combined, these estimates indicate that SN 2008ax likely ejected $\sim$ few $\times$ $0.01 M_{\odot}$ of hydrogen, although a full non-LTE spectral analysis is necessary to be more precise. The non-LTE models of James \& Baron (2010) found that the $\mathrm{H} \alpha$ lines of SNe 1999dn and $2000 \mathrm{H}$ could be modeled using $\lesssim 10^{-3} M_{\odot}$ and $\lesssim 0.2 M_{\odot}$ of hydrogen in the outer envelope, respectively.

\subsection{Spectropolarimetry Comparison}

$\mathrm{SNe}$ IIb are surprisingly well represented in the published sample of SN spectropolarimetry in comparison with SNe IIP (Wang \& Wheeler 2008), given their relative rates. Spectropolarimetry of SN 1993J was presented by Trammell et al. (1993b) and Tran et al. (1997), and the data of Trammell et al. (1993b) were subsequently modeled in detail (Höflich 1995; Höflich et al. 1996). These observations were mostly taken at early phases, when the photosphere was in the hydrogen-rich outer envelope, while our SN 2008ax data were taken when the photosphere was starting to recede through the helium core. Maund et al. (2007b) found that the spectropolarimetry of SN 2001ig changed dramatically at a similar phase. We have compared our day 9 spectropolarimetry to SN 1993J (Tran et al. 1997) in Figure 20. The epoch of the SN 1993J observations was chosen to roughly match the strength of the He I lines with SN 2008ax so that the photosphere would be at an approximately similar depth with respect to the helium-rich core.

The first thing to note from the comparison in Figure 20 is that the polarization near $6600 \AA$ in the peak of the $\mathrm{H} \alpha$ line is almost zero in SN 2008ax, as discussed above, but is $\sim 0.7 \%$ in 
SN 1993J. This reflects the different assumptions used by Tran et al. (1997) to correct their SN 1993J data for ISP. Choosing a different ISP for SN 1993J could lower the overall level of polarization, including the amplitudes of the peaks at the line features, and thus the inferred level of asphericity (Höflich et al. 1996). The line features in the SN 2008ax data are already clearly stronger than in SN 1993J, particularly at $\mathrm{H} \alpha$ and the $\mathrm{H} \beta / \mathrm{Fe}$ II blend.

Wang et al. (2001) found that their spectra and spectropolarimetry of the SN IIb $1996 \mathrm{cb}$ closely resembled those of SN 1993J and proposed that the objects had similar geometries oriented similarly with respect to our line of sight. They wondered if SNe IIb were a homogeneous subclass and whether those objects would have appeared differently from a different viewing angle. Leonard \& Filippenko (2005) presented a single epoch of spectropolarimetry of the SN IIb 2003ed and found that it was also broadly similar to SN 1993J. The SN 2001ig data of Maund et al. (2007b) have some similarity to SN 1993J, but are more distinct than the other objects.

To the extent that our spectropolarimetry of SN 2008ax resembles that of SN 1993J, it may be a result of the same basic phenomenon (overlapping inverted P-Cygni line polarization profiles) in objects with similar spectral features. Thus, the similarity between the spectropolarimetry of SNe 1993J and 1996cb noted by Wang et al. (2001) may simply be a consequence of the close spectroscopic resemblance between those two objects (Qiu et al. 1999). SN 2001ig was quite spectroscopically distinct from SN 1993J (Maund et al. 2007b; Silverman et al. 2009) and had somewhat different polarimetry. As shown in Section 3, SN 2008ax is the least spectroscopically similar of these objects, and so the fact that it has the most distinct polarimetry might have been expected. Furthermore, the speculation of Wang et al. (2001) about the appearance of SNe IIb from different lines of sight will be directly tested in one case due to the discovery of light echoes (Krause et al. 2005; Rest et al. 2008) around the Galactic SN IIb Cas A (Krause et al. 2008), which have enabled the study of that explosion from different vantage points (Rest et al. 2011). If the high $\mathrm{H} \alpha$ polarization we see in SN 2008ax is due to large clumps or a special geometry, the appearance of the $\mathrm{H} \alpha$ line could be quite different along other lines of sight. Indeed, Rest et al. (2011) have found that the $\mathrm{H} \alpha$ velocity of the Cas A SN was higher along one line of sight compared to others that they studied.

Our analysis of the full spectral evolution of SN 2008ax (Section 3) found that the object was more similar to normal $\mathrm{SNe} \mathrm{Ib}$ than to SN 1993J, so it is of interest to compare the spectropolarimetry of those objects as well. Unfortunately, $\mathrm{SNe} \mathrm{Ib}$ are not as well represented in the literature. After the first detection of possible intrinsic $\mathrm{SN}$ polarization in the SN Ib 1983N (McCall 1985), the next SNe Ib observed spectropolarimetrically were $\mathrm{SNe} 1997 \mathrm{dq}$ and 1998T, both of which had substantial $(P>1 \%)$ line-polarization features (Leonard et al. 2000). However, none of those observations have been published. The only comparison $\mathrm{SNe} \mathrm{Ib}$ are the unique SN Ib/c 2005bf (Maund et al. 2007a; Tanaka et al. 2009) and SN 2008D (Maund et al. 2009).

The SN Ib/c 2005bf had many unusual and unique photometric and spectroscopic properties (Tominaga et al. 2005; Folatelli et al. 2006; Parrent et al. 2007; Maeda et al. 2007). However, our automated comparisons using SNID in Section 3 found it to be the closest spectral match to SN 2008ax near maximum light. Single epochs of spectropolarimetry were obtained six days before (Maund et al. 2007a) and eight days after (Tanaka et al.
2009) maximum light. The early observations of Maund et al. (2007a) show very large polarizations in the troughs of the Ca II H\&K and NIR triplet absorptions $(P \lesssim 4 \%)$, with smaller $(P \lesssim 1 \%$ ) features present at the He I $\lambda 5876$ and Fe II lines. The line features decreased in strength by the time of the observations of Tanaka et al. (2009), but at both epochs $q-u$ loops were observed in the He I and Ca II lines.

One result common to the spectropolarimetry of SN 2005bf (Maund et al. 2007a; Tanaka et al. 2009) and SN 2008D (Maund et al. 2009) is that the line features did not share an axis of symmetry. The loops observed in almost all well-detected lines show that the individual features have velocity-dependent angle rotations, but the overall range of angles occupied by the lines is different for different species. Even the same lines observed at different epochs show rotations as the photosphere recedes. In particular, the He I, Ca II, and Fe II lines show misaligned polarization angles in both objects. If the polarization feature near $4700 \AA$ in our day 9 data is mostly due to Fe II, then our SN 2008ax data show exactly the same phenomenon (Figure 14). Interestingly, our He I $\lambda 5876$ data are largely aligned with both the continuum and line-polarization angles of $\mathrm{H} \alpha$ and oxygen at that date.

The continuum polarization we measure on day $9(P=$ $0.64 \pm 0.02 \%)$ is higher than that determined by Maund et al. (2009) for SN 2008D $(P \approx 0.2 \%)$ and Maund et al. (2007a) for SN 2005 bf at early times $(P \approx 0.45 \%)$, although Tanaka et al. (2009) show that different ISP assumptions for SN 2005bf could increase that value to $\sim 1.2 \%$. In the context of the oblate spheroidal models of Höflich (1991), our SN 2008ax continuum polarization implies that the photosphere on day 9 has a minimum axis ratio of about 1.15:1. If we take the polarization in our reddest continuum window on day $99(1.28 \%)$ as representative of the late-time continuum photosphere, then the minimum asphericity in the core rose to $\sim 35 \%$.

Also of interest to us is the evidence presented by Maund et al. (2007a) of only small polarization increases $(P \lesssim$ $0.2 \%$ ) associated with the high-velocity Balmer absorptions in SN 2005bf. While our early-time spectropolarimetry of SN 2008ax showed very large polarization modulations across $\mathrm{H} \alpha$, we were unable to obtain spectropolarimetry near maximum light. At that time, the $\mathrm{H} \alpha$ line was significantly weaker and SN 2008ax resembled normal SNe Ib with a strong $6300 \AA$ feature. An interesting question for future research is the time evolution of polarization modulations at $\mathrm{H} \alpha$ in other stripped-envelope $\mathrm{SNe}$. If the spectroscopic similarities between SN 2008ax and SN 2005bf are meaningful, then perhaps future $\mathrm{SNe} \mathrm{Ib}$ observed near maximum light will also show weak $\mathrm{H} \alpha$ polarization even if the line were strongly polarized at early times, due to the decreased photospheric covering fraction or optical depth at late times. In this context, it is interesting to note that Maund et al. (2009) found a peak in the polarization $(P=0.73 \pm 0.13 \%)$ of SN $2008 \mathrm{D}$ at $6260 \AA$, which they observed was at a rather red wavelength to be associated with the Si II $\lambda 6355$ absorption and tentatively suggested that it instead may have been due to weak high-velocity $\mathrm{H} \alpha$ absorption at $v \approx-17,050 \mathrm{~km} \mathrm{~s}^{-1}$.

\subsection{SN 2008ax as a Supernova Remnant}

The nebular-phase oxygen line profiles presented in Section 3.4 are asymmetric and some lines show multiple peaks. In particular, the double-peaked structure of the $\left.\mathrm{Mg}_{\mathrm{I}}\right] \lambda 4571$ emission line is strong evidence against the possibility of a 
largely blueshifted inner oxygen distribution, which was one possibility explored by Milisavljevic et al. (2010). Instead, the multi-peaked line profiles are likely a sign that models which invoke a clumpy inner oxygen distribution are more correct. Clumping due to Rayleigh-Taylor instabilities in the explosion (e.g., Müller et al. 1991) has been invoked in the past to explain the multi-peaked line profiles observed in other strippedenvelope SNe, including SN 1993J (Matheson et al. 2000b). The continuum polarization of $1.28 \%$ observed in the early nebular phase is also a sign that the inner ejecta of SN 2008ax were highly aspherical. Spectral modeling is necessary to determine how much of the polarization we measure at late times is due to global asphericities versus clumping in the ejecta.

Our spectra of SN 2008ax as it entered the nebular phase show the strong oxygen and calcium lines expected of an object that will develop into an oxygen-rich SN remnant, while our earliest optical spectra demonstrated that a small amount of hydrogen was present in the outer layers of the ejecta. These properties are strongly reminiscent of the Galactic SN remnant Cas A (Chevalier \& Kirshner 1978, 1979). Fesen et al. (1987) identified fast-moving nitrogen-rich ejecta knots that probably represent the outermost layers of the progenitor, which apparently were composed of a small amount of hydrogen, much like a WN star, and similar to one of the potential progenitor models for SN 2008ax. The discovery by Krause et al. (2008) that the spectrum of the $\mathrm{SN}$ explosion giving rise to Cas A was similar to that of SN 1993J only strengthens the relevance of SN 2008ax to Cas A. Similarly, Tominaga et al. (2005) identified Cas A as a possible analog for SN 2005bf. However, Chevalier \& Soderberg (2010) identified Cas A as a member of the SN 1993J-like SNe IIb with extended progenitors, while SN 2008ax was a representative of their class of SNe IIb from compact progenitors.

Optical and X-ray studies of emission from the ejecta of Cas A have shown dramatic compositional inhomogeneities on all scales, from small optical knots (Chevalier \& Kirshner 1978) to the full remnant (Hwang et al. 2004). Iron-rich ejecta from the core have penetrated and overturned the silicon-rich layer that was situated above them in the progenitor (Hughes et al. 2000), while not penetrating the overlying oxygen-rich ejecta (Fesen et al. 2006). The overall appearance of the remnant is very aspherical and turbulent, with silicon-rich "jets" (Fesen 2001; Hwang et al. 2004).

The lack of a simple, well-defined symmetry axis in our earlytime spectropolarimetry of SN 2008ax, along with the differing polarization angles associated with different chemical elements and the multi-peaked nebular line profiles, appears unsurprising in the context of the complex morphology of Cas A. We note that some evolution after explosion has occurred in that remnant due to deceleration by the surrounding gas (Fesen et al. 2006), and possibly also by energy input from radioactive decay (Hughes et al. 2000). Cas A should give pause to anyone attempting to interpret the geometry of distant $\mathrm{SN}$ explosions such as SN 2008ax in a smooth, quasi-spherical or axially symmetric fashion. Wheeler et al. (2008) have attempted to piece together a coherent picture of the Cas A observations in the context of a jet-induced explosion.

\section{CONCLUSIONS}

This detailed study of SN 2008ax was made possible by the luck of a robotic SN search taking an image of the nearby host galaxy only hours after shock breakout from the SN (Mostardi et al. 2008), with the further luck that the image obtained by another observer was able to bracket the time of explosion so tightly (Arbour 2008). This is reminiscent of the fortuitous discovery of the X-ray burst accompanying SN 2008D (Berger \& Soderberg 2008), which allowed numerous groups to obtain detailed follow-up observations of that SN Ib starting soon after explosion (Soderberg et al. 2008; Modjaz et al. 2009; Mazzali et al. 2008; Malesani et al. 2009). Only with the early discovery of SNe can we hope to obtain crucial spectral information about the outer atmospheres of their progenitor stars.

Our first spectrum of SN 2008ax, taken only two days after shock breakout, is one of the earliest spectra of an SN ever obtained and does not resemble any of the spectra of other core-collapse SNe observed at such early times. Strong and deep Balmer absorptions were present, extending to very high velocities $\left(>33,000 \mathrm{~km} \mathrm{~s}^{-1}\right)$. We have presented an extensive sequence of optical and NIR spectra of SN 2008ax in Section 3. Our optical spectra were taken with a relatively high cadence during the first week after explosion and capture the very rapid spectroscopic evolution at early times. The progenitor of SN 2008ax had only a very low-mass hydrogen envelope, so the Balmer lines faded on the rise to maximum light and optical He I lines started to become prominent (see also P08). This spectroscopic transition defines SNe IIb. Our NIR spectra, starting only 10 days after shock breakout, showed little evidence of hydrogen and were dominated by the helium lines expected in a $\mathrm{SN} \mathrm{Ib.}$

Branch et al. (2002) found that the spectra of many SNe Ib exhibited an absorption near $6300 \AA$ and presented arguments in favor of an identification with $\mathrm{H} \alpha$. Our early optical spectra of SN 2008ax show unambiguous Balmer lines, with the $\mathrm{H} \alpha$ absorption smoothly evolving into an absorption near $6270 \AA$. By maximum light, the optical-through-NIR spectra very closely resembled those of the SN Ib 1999ex. If the first spectrum of SN 2008ax had not been taken until maximum light or shortly afterward, as is typical of most SNe, it likely would have been classified as an SN Ib with some hydrogen absorption, as were a few of our comparison objects (Figure 7).

This suggests that the distinction between $\mathrm{SNe} \mathrm{IIb}$ and $\mathrm{Ib}$ may in part depend on the quality and timing of spectroscopic observations. The case of SN 1993J was not ambiguous because that object had a sufficiently thick hydrogen envelope for $\mathrm{H} \alpha$ to be clearly present at all times. At the other extreme, the SN Ib 2008D was observed very shortly after explosion and never showed the strong Balmer absorptions seen in SN 2008ax (Figure 4). However, most of the other objects plotted in Figure 7 appear to be intermediate between those two, and the distinction between those objects spectroscopically classified as $\mathrm{SNe} \mathrm{Ib}$ and as $\mathrm{SNe}$ IIb may not be physically meaningful. If the $\mathrm{SNe} \mathrm{Ib}$ had been observed as soon after explosion as SN 2008ax, then many of them likely would also have been classified as SNe IIb. Our NIR spectral sequence of SN 2008ax (Figure 3) and the radio light curves of Roming et al. (2009) also support a closer correspondence of SN 2008ax to typical SNe Ib than to SN 1993J, despite the prominence of optical hydrogen lines at early times.

These observations of SN 2008ax support the suggestion of Branch et al. (2002) that the spectra of objects classified as $\mathrm{SNe} \mathrm{Ib}$ frequently exhibit some $\mathrm{H} \alpha$ absorption. Therefore, the progenitors of many $\mathrm{SNe} \mathrm{Ib}$ must explode with some hydrogen in their outer envelopes. The processes that consistently lead to such a small, but nonzero, amount of hydrogen in the outer envelope before explosion are not understood, and represent a challenge for stellar evolution models. Resolution of the ambiguities 
in the direct detection of the progenitor of SN 2008ax (Crockett et al. 2008), along with studies of the progenitors of future stripped-envelope $\mathrm{SNe}$, will help clarify the situation.

Our three epochs of spectropolarimetry and the nebular line profiles demonstrate that SN 2008ax showed strong asphericities starting shortly after explosion and continuing until the early nebular phase. The $3.4 \%$ polarization feature at $\mathrm{H} \alpha$ on day 9 was unusually strong and may indicate that the appearance of the $\mathrm{H} \alpha$ absorption could depend on the viewing orientation with respect to the object. After correction for ISP, the hydrogen, helium, and oxygen distributions were found to be largely aligned with the continuum axis of symmetry, while the iron and calcium lines exhibited strong deviations from this axial symmetry. All lines with sufficiently good detections also showed loops in the $q-u$ plane indicative of velocity-dependent angle rotations of unknown origin. These deviations from axisymmetry appear to be common in stripped-envelope $\mathrm{SNe}$, but their cause is mysterious. Detailed spectral synthesis, including polarization, of explosion models is necessary to understand whether those models are capable of reproducing these observations.

We acknowledge M. Ganeshalingam, C. V. Griffith, J. Hodge, K. Merriman, R. Mostardi, D. Poznanski, and X. Wang for assistance with some of the observations. We would also like to thank the observers of the Lick AGN Monitoring Project 2008 (LAMP) for obtaining some of these SN 2008ax spectra during the nightly SN observations, in particular A. J. Barth, V. N. Bennert, M. C. Bentz, G. Canalizo, K. D. Hiner, C. E. Thornton, J. L. Walsh, and J.-H. Woo. Some of the data presented herein were obtained at the W. M. Keck Observatory, which is operated as a scientific partnership among the California Institute of Technology, the University of California, and the National Aeronautics and Space Administration; it was made possible by the generous financial support of the W. M. Keck Foundation. The authors wish to recognize and acknowledge the very significant cultural role and reverence that the summit of Mauna Kea has always had within the indigenous Hawaiian community; we are most fortunate to have the opportunity to conduct observations from this mountain. We also would like to thank the expert assistance of the Keck and Lick staffs in making these observations possible. Data for this manuscript were obtained by Visiting Astronomers at the Infrared Telescope Facility, which is operated by the University of Hawaii under Cooperative Agreement No. NNX-08AE38A with the National Aeronautics and Space Administration, Science Mission Directorate, Planetary Astronomy Program. We thank Alan Tokunaga and the IRTF staff for their help performing timely observations. A.V.F.'s supernova research group at UC Berkeley has been supported by NSF grants AST-0607485 and AST-0908886, as well as by the TABASGO Foundation. M.M. is supported by a fellowship from the Miller Institute for Basic Research in Science. D.K.L., R.J.R., and R.W.R. were supported by The Aerospace Corporation's Independent Research and Development program.

Facilities: Shane (Kast), Keck:I (LRIS, HIRES), Keck:II (ESI), IRTF (SpeX)

\section{APPENDIX: ISM PROPERTIES OF NGC 4490}

High-resolution spectroscopy of SNe can also be used as a tool to study the ISM of SN host galaxies. Our HIRES spectra of SN 2008ax reveal absorptions from a number of DIBs (Herbig 1975), as well as the Na I and KI lines shown in Figure 2. The absorbers responsible for DIBs are still largely unknown (e.g., Herbig 1995), so determining the presence and relative strengths

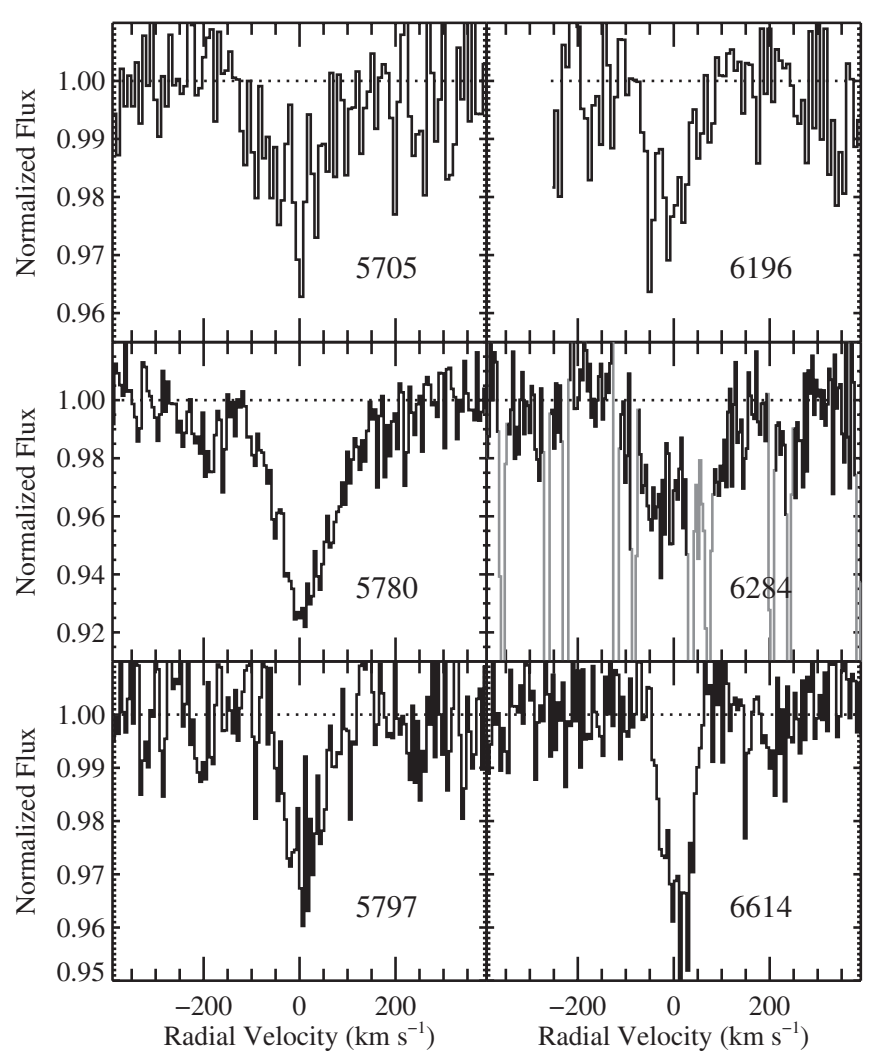

Figure 21. DIBs in NGC 4490. Each panel is labeled with the wavelength of the DIB feature. The $\lambda 6284$ DIB is heavily contaminated by telluric absorption. The narrow telluric absorption features in that panel are grayed out and there is still significant uncertainty in the continuum level.

Table 4

ISM Absorption Features in NGC 4490

\begin{tabular}{lcrcr}
\hline \hline Absorber & $\begin{array}{c}\text { Wavelength } \\
(\AA)\end{array}$ & $\begin{array}{c}\text { EW } \\
(\mathrm{m} \AA)\end{array}$ & $\begin{array}{c}\text { Uncertainty }^{\mathrm{a}} \\
(\mathrm{m} \AA)\end{array}$ & $\begin{array}{r}\mathrm{MW}^{\mathrm{b}} \\
(\mathrm{m} \AA)\end{array}$ \\
\hline DIB & 5705 & 61.8 & 8.4 & 88.5 \\
DIB & 5780 & 228.0 & 7.0 & 289.5 \\
DIB & 5797 & 44.6 & 6.5 & 66.0 \\
DIB & 6196 & 52.1 & 10.3 & 30.5 \\
DIB & 6614 & 53.3 & 7.6 & 115.5 \\
Na I & 5890 & 959.4 & 1.9 & $\ldots$ \\
Na I & 5896 & 827.4 & 2.0 & $\ldots$ \\
K I & 7699 & 142.0 & 6.2 & $\ldots$ \\
\hline
\end{tabular}

Notes.

${ }^{a}$ Not including uncertainty in continuum placement.

b Average value for a Galactic line of sight from Jenniskens \& Désert (1994), scaled to our value of $E(B-V)=0.5 \mathrm{mag}$.

of the absorptions in environments far removed from the local conditions of the ISM in the Galaxy may provide significant clues. The smooth continua of bright $\mathrm{SNe}$ provide a good backdrop to measure the otherwise hard-to-study properties of DIBs in external galaxies. DIB features have been measured in a handful of SNe so far (e.g., Rich 1987; Sollerman et al. 2005; Cox \& Patat 2008). Most of the objects which have been studied to date are SNe Ia. A core-collapse SN such as SN 2008ax has the potential to probe different ISM conditions due to its likely proximity to the sites of massive-star formation.

An in-depth study of DIB features in NGC 4490 is beyond the scope of this work. However, we have plotted some prominent DIBs in Figure 21 and measured their EWs in Table 4 for 
the benefit of other researchers. The measured EWs for the atomic lines are integrated over $\pm 40 \mathrm{~km} \mathrm{~s}^{-1}$ around our adopted value for the recession velocity of SN 2008ax of $630 \mathrm{~km} \mathrm{~s}^{-1}$. The EWs of the DIBs are integrated over $\pm 200 \mathrm{~km} \mathrm{~s}^{-1}$. The DIB near $\lambda 5705$ is actually a blend of two features at $5704.75 \AA$ and $5705.13 \AA$ in the compilation of Jenniskens $\&$ Désert (1994), but we have made no effort to decompose the observed absorption. Strong telluric absorption severely hampered continuum placement around $\lambda 6284$ and made any measurement of the EW unreliable, so we have not reported a value. The EWs of the DIBs seen in an average Milky Way cloud with the same $E(B-V)$ value as SN 2008ax (Jenniskens $\&$ Désert 1994) are also listed in Table 4 . The $\lambda 6614$ absorption seems relatively weak compared to the average Galactic line of sight, an effect that was also seen along the line of sight to SN 2006X in M100 by Cox \& Patat (2008).

\section{REFERENCES}

Aldering, G., Humphreys, R. M., \& Richmond, M. 1994, AJ, 107, 662 Arbour, R. 2008, CBET, 1286, 2

Baron, E., Branch, D., Hauschildt, P. H., et al. 2000, ApJ, 545, 444 Baron, E., Hauschildt, P. H., Branch, D., et al. 1995, ApJ, 441, 170 Benetti, S., Cappellaro, E., Turatto, M., \& Pastorello, A. 2000, IAU Circ., 7375, 1

Benetti, S., Patat, F., Turatto, M., et al. 1994, A\&A, 285, L13

Berger, E., \& Soderberg, A. M. 2008, GRB Coord. Netw., 7159, 1

Blondin, S., Filippenko, A. V., Foley, R. J., et al. 2008, CBET, 1285, 1

Blondin, S., \& Tonry, J. L. 2007, ApJ, 666, 1024

Branch, D., Benetti, S., Kasen, D., et al. 2002, ApJ, 566, 1005

Chevalier, R. A., \& Kirshner, R. P. 1978, ApJ, 219, 931

Chevalier, R. A., \& Kirshner, R. P. 1979, ApJ, 233, 154

Chevalier, R. A., \& Soderberg, A. M. 2010, ApJ, 711, L40

Chornock, R., Filippenko, A. V., Li, W., et al. 2008, CBET, 1298, 1

Chornock, R., Filippenko, A. V., Li, W., \& Silverman, J. M. 2010, ApJ, 713, 1363

Chugai, N. N. 1992, Sov. Astron. Lett., 18, 168

Chugai, N. N., \& Danziger, I. J. 2003, Astron. Lett., 29, 649

Clocchiatti, A., Suntzeff, N. B., Phillips, M. M., et al. 2001, ApJ, 553, 886

Clocchiatti, A., Wheeler, J. C., Benetti, S., \& Frueh, M. 1996, ApJ, 459, 547

Cox, N. L. J., \& Patat, F. 2008, A\&A, 485, L9

Crockett, R. M., Eldridge, J. J., Smartt, S. J., et al. 2008, MNRAS, 391, L5

Cropper, M., Bailey, J., McCowage, J., Cannon, R. D., \& Couch, W. J. 1988, MNRAS, 231, 695

Cushing, M. C., Vacca, W. D., \& Rayner, J. T. 2004, PASP, 116, 362

Deng, J. S., Qiu, Y. L., Hu, J. Y., Hatano, K., \& Branch, D. 2000, ApJ, 540, 452

Dessart, L., Blondin, S., Brown, P. J., et al. 2008, ApJ, 675, 644

Elmhamdi, A., Danziger, I. J., Branch, D., et al. 2006, A\&A, 450, 305

Fesen, R. A. 2001, ApJS, 133, 161

Fesen, R. A., Becker, R. H., \& Blair, W. P. 1987, ApJ, 313, 378

Fesen, R. A., Hammell, M. C., Morse, J., et al. 2006, ApJ, 636, 859

Filippenko, A. V. 1988, AJ, 96, 1941

Filippenko, A. V. 1997, ARA\&A, 35, 309

Filippenko, A. V., Matheson, T., \& Barth, A. J. 1994, AJ, 108, 2220

Filippenko, A. V., Matheson, T., \& Ho, L. 1993, ApJ, 415, L103

Folatelli, G., Contreras, C., Phillips, M. M., et al. 2006, ApJ, 641, 1039

Fransson, C., \& Chevalier, R. A. 1989, ApJ, 343, 323

Garnavich, P. M., \& Ann, H. B. 1994, AJ, 108, 1002

Gerardy, C. L., Fesen, R. A., Marion, G. H., et al. 2004, in Cosmic Explosions in Three Dimensions, ed. P. Höflich, P. Kumar, \& J. C. Wheeler (Cambridge: Cambridge Univ. Press), 57

Hamuy, M., Deng, J., Mazzali, P. A., et al. 2009, ApJ, 703, 1612

Hamuy, M., Maza, J., Pinto, P. A., et al. 2002, AJ, 124, 417

Heger, A., Fryer, C. L., Woosley, S. E., Langer, N., \& Hartmann, D. H. 2003, ApJ, 591,288

Herbig, G. H. 1975, ApJ, 196, 129

Herbig, G. H. 1995, ARA\&A, 33, 19

Höflich, P. 1991, A\&A, 246, 481

Höflich, P. 1995, ApJ, 440, 821

Höflich, P., Wheeler, J. C., Hines, D. C., \& Trammell, S. R. 1996, ApJ, 459, 307

Houck, J. C., \& Fransson, C. 1996, ApJ, 456, 811

Howell, D. A., Höflich, P., Wang, L., \& Wheeler, J. C. 2001, ApJ, 556, 302
Hughes, J. P., Rakowski, C. E., Burrows, D. N., \& Slane, P. O. 2000, ApJ, 528, L109

Hunter, D. J., Valenti, S., Kotak, R., et al. 2009, A\&A, 508, 371

Hwang, U., Laming, J. M., Badenes, C., et al. 2004, ApJ, 615, L117

James, S., \& Baron, E. 2010, ApJ, 718, 957

Jeffery, D. J. 1989, ApJS, 71, 951

Jeffery, D. J. 1991a, ApJ, 375, 264

Jeffery, D. J. 1991b, ApJS, 77, 405

Jenniskens, P., \& Désert, F.-X. 1994, A\&AS, 106, 39

Kasen, D., Nugent, P., Wang, L., et al. 2003, ApJ, 593, 788

Krause, O., Birkmann, S. M., Usuda, T., et al. 2008, Science, 320, 1195

Krause, O., Rieke, G. H., Birkmann, S. M., et al. 2005, Science, 308, 1604

Leonard, D. C., \& Filippenko, A. V. 2005, in ASP Conf. Ser. 342, 1604-2004: Supernovae as Cosmological Lighthouses, ed. M. Turatto et al. (San Francisco, CA: ASP), 330

Leonard, D. C., Filippenko, A. V., Ardila, D. R., \& Brotherton, M. S. 2001, ApJ, 553,861

Leonard, D. C., Filippenko, A. V., Ganeshalingam, M., et al. 2006, Nature, 440, 505

Leonard, D. C., Filippenko, A. V., Gates, E. L., et al. 2002, PASP, 114, 35

Leonard, D. C., Filippenko, A. V., \& Matheson, T. 2000, in AIP Conf. Ser. 522, Cosmic Explosions, ed. W. W. Zhang \& S. S. Holt (Melville, NY: AIP), 165 Lewis, J. R., Walton, N. A., Meikle, W. P. S., et al. 1994, MNRAS, 266, L27

Li, W., van Dyk, S. D., Cuillandre, J.-C., et al. 2008, CBET, 1290, 1

Li, W., Leaman, J., Chornock, R., et al. 2011, MNRAS, 412, 1441

Lucy, L. B. 1991, ApJ, 383, 308

Maeda, K., Kawabata, K., Mazzali, P. A., et al. 2008, Science, 319, 1220

Maeda, K., Tanaka, M., Nomoto, K., et al. 2007, ApJ, 666, 1069

Malesani, D., Fynbo, J. P. U., Hjorth, J., et al. 2009, ApJ, 692, L84

Marion, G. H., Höflich, P., Gerardy, C. L., et al. 2009, AJ, 138, 727

Marion, H., Garnavich, P., Gerardy, C. L., et al. 2008, CBET, 1305, 1

Martí-Vidal, I., Marcaide, J. M., Alberdi, A., et al. 2009, A\&A, 499, 649

Matheson, T., Filippenko, A. V., Barth, A. J., et al. 2000a, AJ, 120, 1487

Matheson, T., Filippenko, A. V., Ho, L. C., Barth, A. J., \& Leonard, D. C. 2000b, AJ, 120, 1499

Matheson, T., Filippenko, A. V., Li, W., Leonard, D. C., \& Shields, J. C. 2001, AJ, 121,1648

Matthews, K., Neugebauer, G., Armus, L., \& Soifer, T. A. 2002, AJ, 123, 753

Matzner, C. D., \& McKee, C. F. 1999, ApJ, 510, 379

Maund, J. R., \& Smartt, S. J. 2009, Science, 324, 486

Maund, J. R., Smartt, S. J., Kudritzki, R. P., Podsiadlowski, P., \& Gilmore, G. F. 2004, Nature, 427, 129

Maund, J. R., Wheeler, J. C., Baade, D., et al. 2009, ApJ, 705, 1139

Maund, J. R., Wheeler, J. C., Patat, F., et al. 2007a, MNRAS, 381, 201

Maund, J. R., Wheeler, J. C., Patat, F., et al. 2007b, ApJ, 671, 1944

Maurer, I., Mazzali, P. A., Taubenberger, S., \& Hachinger, S. 2010a, MNRAS, 409, 1441

Maurer, J. I., Mazzali, P. A., Deng, J., et al. 2010b, MNRAS, 402, 161

Mazzali, P., Kawabata, K. S., Maeda, K., et al. 2005, Science, 308, 1284

Mazzali, P., Valenti, S., Della Valle, M., et al. 2008, Science, 321, 1185

Mazzali, P. A., Deng, J., Hamuy, M., \& Nomoto, K. 2009, ApJ, 703, 1624

McCall, M. L. 1985, in Supernovae as Distance Indicators, ed. N. Bartel (Lect. Notes Phys., Vol. 224; Berlin: Springer), 48

Meikle, W. P. S., Allen, D. A., Spyromilio, J., \& Varani, G.-F. 1989, MNRAS, 238, 193

Menzies, J. W., Catchpole, R. M., van Vuuren, G., et al. 1987, MNRAS, 227, 39P

Milisavljevic, D., Fesen, R., Gerardy, C., Kirshner, R., \& Challis, P. 2010, ApJ, 709, 1343

Miller, J. S., Robinson, L. B., \& Goodrich, R. W. 1988, in Instrumentation for Ground-based Astronomy, ed. L. B. Robinson (New York: Springer), 157

Miller, J. S., \& Stone, R. P. S. 1993, Lick Observatory Technical Reports, No. 66 (Santa Cruz, CA: Lick Obs.)

Modjaz, M., Kirshner, R. P., Blondin, S., Challis, P., \& Matheson, T. 2008, ApJ, 687, L9

Modjaz, M., Li, W., Butler, N., et al. 2009, ApJ, 702, 226

Mostardi, R., Li, W., \& Filippenko, A. V. 2008, CBET, 1280, 1

Müller, E., Fryxell, B., \& Arnett, D. 1991, A\&A, 251, 505

Munari, U., \& Zwitter, T. 1997, A\&A, 318, 269

Nomoto, K., Suzuki, T., Shigeyama, T., et al. 1993, Nature, 364, 507

Oke, J. B., Cohen, J. G., Carr, M., et al. 1995, PASP, 107, 375

Parrent, J., Branch, D., Troxel, M. A., et al. 2007, PASP, 119, 135

Pastorello, A., Kasliwal, M. M., Crockett, R. M., et al. 2008, MNRAS, 389, 955 (P08)

Podsiadlowski, P., Hsu, J. J. L., Joss, P. C., \& Ross, R. R. 1993, Nature, 364, 509

Qiu, Y., Li, W., Qiao, Q., \& Hu, J. 1999, AJ, 117, 736 
Quimby, R. M., Wheeler, J. C., Höflich, P., et al. 2007, ApJ, 666, 1093

Rayner, J. T., Toomey, D. W., Onaka, P. M., et al. 2003, PASP, 115, 362

Rest, A., Foley, R. J., Sinnott, B., et al. 2011, ApJ, 732, 3

Rest, A., Welch, D. L., Suntzeff, N. B., et al. 2008, ApJ, 681, L81

Rich, R. M. 1987, AJ, 94, 651

Richmond, M. W., Treffers, R. R., Filippenko, A. V., et al. 1994, AJ, 107, 1022

Roming, P. W. A., Pritchard, T. A., Brown, P. J., et al. 2009, ApJ, 704, L118

Ryder, S. D., Murrowood, C. E., \& Stathakis, R. A. 2006, MNRAS, 369, L32

Ryder, S. D., Sadler, E. M., Subrahmanyan, R., et al. 2004, MNRAS, 349, 1093

Sauer, D. N., Mazzali, P. A., Deng, J., et al. 2006, MNRAS, 369, 1939

Schlegel, D. J., Finkbeiner, D. P., \& Davis, M. 1998, ApJ, 500, 525

Schmidt, B. P., Kirshner, R. P., Eastman, R. G., et al. 1993, Nature, 364, 600

Schmidt, G. D., Elston, R., \& Lupie, O. L. 1992, AJ, 104, 1563

Serkowski, K., Mathewson, D. S., \& Ford, V. L. 1975, ApJ, 196, 261

Shapiro, P. R., \& Sutherland, P. G. 1982, ApJ, 263, 902

Sheinis, A. I., Bolte, M., Epps, H. W., et al. 2002, PASP, 114, 851

Shigeyama, T., Suzuki, T., Kumagai, S., et al. 1994, ApJ, 420, 341

Silverman, J. M., Mazzali, P., Chornock, R., et al. 2009, PASP, 121, 689

Smartt, S. J., Eldridge, J. J., Crockett, R. M., \& Maund, J. 2009, MNRAS, 395, 1409

Smith, N., \& Conti, P. S. 2008, ApJ, 679, 1467

Smith, N., Li, W., Filippenko, A. V., \& Chornock, R. 2011, MNRAS, 412, 1522

Soderberg, A. M., Berger, E., Page, K. L., et al. 2008, Nature, 453, 469

Soderberg, A. M., Chevalier, R. A., Kulkarni, S. R., \& Frail, D. A. 2006, ApJ, 651,1005

Sollerman, J., Cox, N., Mattila, S., et al. 2005, A\&A, 429, 559

Stritzinger, M., Hamuy, M., Suntzeff, N. B., et al. 2002, AJ, 124, 2100

Stritzinger, M., Mazzali, P., Phillips, M. M., et al. 2009, ApJ, 696, 713
Swartz, D. A., Clocchiatti, A., Benjamin, R., Lester, D. F., \& Wheeler, J. C. 1993, Nature, 365, 232

Tanaka, M., Kawabata, K. S., Maeda, K., et al. 2009, ApJ, 699, 1119

Taubenberger, S., Navasardyan, H., Maurer, J. I., et al. 2011, MNRAS, 413, 2140

Taubenberger, S., Pastorello, A., Mazzali, P. A., et al. 2006, MNRAS, 371, 1459

Taubenberger, S., Valenti, S., Benetti, S., et al. 2009, MNRAS, 397, 677

Tominaga, N., Tanaka, M., Nomoto, K., et al. 2005, ApJ, 633, L97

Trammell, S. R., Dinerstein, H. L., \& Goodrich, R. W. 1993a, ApJ, 402, 249

Trammell, S. R., Hines, D. C., \& Wheeler, J. C. 1993b, ApJ, 414, L21

Tran, H. D. 1995, ApJ, 440, 578

Tran, H. D., Filippenko, A. V., Schmidt, G. D., et al. 1997, PASP, 109, 489

Tsvetkov, D. Y., Volkov, I. M., Baklanov, P., Blinnikov, S., \& Tuchin, O. 2009, Perem. Zvezdy, 29, 2

Vacca, W. D., Cushing, M. C., \& Rayner, J. T. 2003, PASP, 115, 389

Valenti, S., Elias-Rosa, N., Taubenberger, S., et al. 2008, ApJ, 673, L155

Vogt, S. S., Allen, S. L., Bigelow, B. C., et al. 1994, Proc. SPIE, 2198, 362

Walker, A. R., \& Suntzeff, N. B. 1990, PASP, 102, 131

Wang, L., Howell, D. A., Höflich, P., \& Wheeler, J. C. 2001, ApJ, 550, 1030

Wang, L., \& Wheeler, J. C. 2008, ARA\&A, 46, 433

Wheeler, J. C., \& Filippenko, A. V. 1996, in IAU Colloq. 145, Supernovae and Supernova Remnants, ed. R. McCray \& Z. Wang (Cambridge: Cambridge Univ. Press), 241

Wheeler, J. C., Barker, E., Benjamin, R., et al. 1993, ApJ, 417, L71

Wheeler, J. C., Maund, J. R., \& Couch, S. M. 2008, ApJ, 677, 1091

Whittet, D. C. B., Martin, P. G., Hough, J. H., et al. 1992, ApJ, 386, 562

Woosley, S. E., Eastman, R. G., Weaver, T. A., \& Pinto, P. A. 1994, ApJ, 429, 300

Woosley, S. E., Pinto, P. A., Martin, P. G., \& Weaver, T. A. 1987, ApJ, 318, 664 